\title{
$2251+158$ (3C 454.3): detection of an arc-like structure on parsec scales ${ }^{\star}$
}

\author{
S. Britzen ${ }^{1}$, Shan-Jie Qian' ${ }^{2}$, A. Witzel ${ }^{1}$, T. P. Krichbaum ${ }^{1}$, M. Aller ${ }^{3}$, H. Aller ${ }^{3}$, O. Kurtanidze ${ }^{4,5}$, \\ S. Vercellone ${ }^{6}$, and G. M. Richter ${ }^{7}$
}

\author{
${ }^{1}$ Max-Planck-Institut für Radioastronomie, Auf dem Hügel 69, 53121 Bonn, Germany \\ e-mail: sbritzen@mpifr.de \\ 2 National Astronomical Observatories, Chinese Academy of Sciences, 100012 Beijing, PR China \\ 3 Department of Astronomy, University of Michigan, Ann Arbor, MI, USA \\ 4 Abastumani Observatory, Mt. Kanobili, Georgia \\ 5 Engelhardt Astronomical Observatory, Kazan Federal University, Tatarstan, Russia \\ ${ }^{6}$ INAF - IASF Palermo, Italy \\ 7 Astrophysikalisches Institut Potsdam, An der Sternwarte 16, 14482 Potsdam, Germany
}

Received 31 July 2012 / Accepted 7 May 2013

\begin{abstract}
Context. $2251+158$ (3C 454.3) is a well-studied quasar with rather unusual properties. It is among the most variable and brightest gamma-ray emitting active galactic nucleus (AGN) in the sky observed by Fermi. The multi-wavelength flux density emission of this source is peculiar, and so is the very long baseline interferometry (VLBI) structure. While it is usually assumed that the jet and jet-components in a given AGN show more or less the same properties (e.g., apparent velocities and paths) with time, we demonstrate here that unusual morphologies (arc-like structure) can occur as temporal phenomena. We also show that the kinematic properties of jet components change from slow to fast apparent motion.

Aims. We present the detection of an arc around the core region. To understand the physical nature of this and other peculiar kinematic properties of the parsec-scale jet of 2251+158 we performed detailed radio-interferometric studies of this prototypical AGN.

Methods. We (re-)analyzed 32 Very Long Baseline Array (VLBA) observations covering 16 years in time (between 1995.57 and 2011.48), observed at $15 \mathrm{GHz}$ from the Monitoring Of Jets in Active galactic nuclei with VLBA Experiments (MOJAVE/2 cm) survey and derived the parameters of the observed VLBI structure. Three $43 \mathrm{GHz}$ VLBA observations from 2001.22, 2005.38, and 2005.76 have been reprocessed. We fitted the components of the VLBI structure with Gaussian components. We studied the properties of light-curves obtained in the radio $(4.8,8.0$, and $14.5 \mathrm{GHz})$ and optical regime $(R$-band), and studied the correlations between the flaring properties and the VLBI structure of the source.

Results. We report the detection of an arc-like structure around the core of an AGN. This ring-structure becomes visible in VLBI maps around 1996. It expands with an apparent proper motion between $0.12 \pm 0.01 \mathrm{mas} \mathrm{yr}^{-1}$ and $0.19 \pm 0.01 \mathrm{mas}^{-1}$ (corresponding to $5.4 \pm 0.3 c$ to $9.0 \pm 0.4 c$ ) and dominates the parsec-scale structure for about 14 years. In addition, we report a significant change in the kinematic properties of jet components close to the core. A phase of slow apparent radial motion (directed away from the core) of two inner jet components (A and B) and no component ejection after a major radio-flare in 1995 is atypical for AGN. This phase is followed by a more typical behaviour in which the same components separate with higher apparent speeds from the core and two new components appear to be ejected from the central region.

Conclusions. We observe significant changes in the morphological and kinematic properties of the parsec-scale jet. Taken together, it is tempting to see a causal connection between the different phenomena. We briefly suggest and discuss several possible physical scenarios to explain the observed phenomena. The kinematic changes as well as the changes in the flaring characteristics could be explained geometrically because of a change in the angle to the line of sight towards the observer assuming a helical structure of the jet. Another possible explanation for the observed phenomena and the correlations among them could be a precessing jet (precession period on timescale of 14.5 years) in combination with its interaction with an external inhomogeneous medium surrounding the core. These and other possible explanations will be investigated further and manuscripts presenting the results are in preparation.
\end{abstract}

Key words. techniques: interferometric - radio continuum: galaxies - quasars: individual: 3C 454.3

\section{Introduction}

$2251+158(3 \mathrm{C} 454.3)$ is a magnitude 16 quasar at a redshift of $z=0.859$. It belongs to the class of optically violent variable sources. The source has been monitored with telescopes from the radio to the TeV-regime in order to unravel the origin of the high energetic radiation detected with AGILE and the

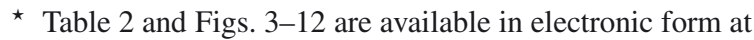
http://www. aanda.org
GLAST/Fermi satellite (e.g., Tosti et al. 2008; Ackermann et al. 2010). $2251+158$ is the most variable and intense gamma-ray blazar detected by AGILE and Fermi during the last couple of years (e.g., Vercellone 2012). Detailed studies have been performed concerning the unusual multi-wavelength flaring of this source (e.g., Raiteri et al. 2011; Bonnoli et al. 2011; Ogile et al. 2011; Wehrle et al. 2012).

The radio morphology has been studied and monitored through many VLBI observations. Some of the VLBI observations are listed here: in the centimeter regime by e.g., 
Moellenbrock et al. (1996), within the VSOP pre-launch survey (e.g., Fomalont et al. 2000), the MOJAVE/2 cm survey (e.g., Lister et al. 2005; Homan et al. 2006), and at higher frequencies by e.g., Lonsdale et al. (1998), Rantakyro et al. (1998), Lobanov et al. (2000), Marscher et al. (2002), and recently by Jorstad et al. $(2010,2012)$. The connection between mm-continuum variations and the VLBI structure has been investigated by e.g., Savolainen et al. (2002). The polarimetry at $43 \mathrm{GHz}$ has been studied by e.g., Kemball et al. (1996). A detailed early study of component motion in the parsec-scale jet of this source has been performed by Pauliny-Toth et al. (1987). Qian et al. (2007) showed that the long-term radio-variability at 4.8 and $8 \mathrm{GHz}$ can be explained by a binary black hole model.

$2251+158$ reveals quite a number of properties which are atypical for AGN and in particular for quasars. To better understand the physical processes in AGN and in this source, we studied in detail the parsec-scale morphology and its evolution over roughly 16 years at several radio frequencies. In addition, we collected light-curve information from the radio and the optical wavebands. By studying not only the morphology, but also the emission processes, we hope to put the pieces together. We expect that all the different properties taken together form a puzzle tat - only when as complete as possible - can tell about the physical origin of the enigmatic properties in this source which at first glance seem to be unrelated.

This paper is organized in the following way: we first describe the VLBA observational data and the data reduction (Sect. 2). We then report on the VLBA results (Sect. 3), the peculiar kinematic properties of the inner jet components (Sect. 3.1), the detection of an arc-like structure (Sect. 3.2), and the for quasars rather typical kinematics of the the outer jet components (Sect. 3.3). We describe in detail the properties of the arc-like structure, and analyze separately this new feature and the underlying jet and its properties. Section 4 describes the multiwavelength radio light-curve-variability, the optical light-curve information, and their analysis. Some of the complex flaring phenomena can be directly related to the observed changes of the kinematics of the VLBI components and the structural changes of the VLBI-jet. In the discussion, we give an overview over the findings and compare them with earlier results reported in the literature. We discuss the possible physical nature of the peculiar kinematics, the arc-like structure and the correlation with the observed flux density variability. We compare the findings for $2251+158$ with those we published for other BL Lac objects and quasars.

Throughout this paper we use $H_{0}=71 \mathrm{~km} \mathrm{~s}^{-1}, \Omega_{\mathrm{m}}=0.27$, $\Omega_{\Lambda}=0.73$.

\section{VLBA observations and model-fitting}

Thirty-two VLBA observations performed within the $2 \mathrm{~cm}$ MOJAVE program between 1995.57 and 2011.48 have been reanalyzed (re-imaged and model-fitted). For all the data sets we performed model fitting of circular Gaussian components within the DIFMAP package (v.2.4b, Shepherd 1997) to the visibility data. In order to find the optimum set of components and parameters, we fitted every data set starting from a point-like model. Circular components have been chosen in order to simplify the component identification and the comparison.

The determination of model-fit parameter uncertainties is a complex topic and no completely satisfying solution to this problem has been presented so far. A detailed discussion of modelfitting uncertainties and their influence on the parameters is presented in Britzen et al. (2008). The uncertainties of each data set in general are influenced by different uv-coverages, calibration, sensitivities, etc. The relative positional errors vary along the jet, and the brightness of the individual component affects the fitted positions as well. We estimate the relative uncertainty in flux density to be 5\% and in FWHM to be $20 \%$. The uncertainty in the position has been determined from the variation in the values derived for different model-fits with different numbers of components for the same epoch. We find that $1 / 5$ of the average beam size represents a reasonable uncertainty estimate for the distance of a component from the core, and 5 degrees is a reasonable estimate for the for the position angle (direction of a component relative to the core) uncertainty. Surely the uncertainty in the position angle from the core depends on the distance of the component away from the core, such that more distant components have more precise measurements of position angle. To trace the evolution of the substructure across the epochs, we list the parameters (epoch, flux density, core separation, position angle, and major axis) of the Gaussian components in Table 2.

The table includes several features marked as " $x$ ". Although these features might represent jet components, an identification was not ambiguously possible. In some cases we performed a flux density weighted averaging when an additional less bright component fit the data best. We assume that in general, on the basis of the available data, two outer components fit the observed outer jet structure best.

In Figs. 3-5 we show the images resulting from the reanalysis (model-fitting) of the $15 \mathrm{GHz}$ Mojave radio data. Superimposed are the Gaussian fits to the data. The images and contours shown are based on the re-analysis and model-fitting process performed by the authors of this paper, and were not taken from the MOJAVE web-page.

The $43 \mathrm{GHz}$ data (Fig. 6) were taken from different VLBA experiments available on the National Radio Astronomy Observatory (NRAO) Data Archive System. All of the data were correlated at NRAO in Socorro, and fringe-fitted and calibrated in the standard manner using AIPS. Clean maps were made using DIFMAP. The analysis of the data sets was done at MPIfR in Bonn.

\section{Results of the VLBI analysis}

We confirm the results concerning the general VLBI structure of $2251+158$ by Pauliny-Toth et al. (1987). The source shows a core-jet structure with a jet extending about 10 mas from the core oriented along an overall position angle of $\sim-50^{\circ}$. Embedded in the jet are several distinct features. Despite the general agreement with earlier studies, the source structure in the epochs analyzed here differs significantly from previous studies when investigated in detail.

In the time period covered by the observations, the structure contains an additional arc-like feature around the core region of the AGN. We show that the complex parsec-scale morphology of $2251+158$ can be decomposed into several substructures: the core $(\mathrm{K})$, the inner jet components (A, B, C, and D), an expanding arc-like feature (represented by R0, R1, R2, R3, and R4), and the outer jet components ( $\mathrm{Y}$ and $\mathrm{Z}$ ) at a distance of $8-10$ mas from the core. In Fig. 1, most of the identified components are shown and labeled. Figure 2 shows a superposition of two epochs of data separated in time by roughly three years. This serves to illustrate the following description of the kinematics of the arc-components better. Figures 1 and 2 were redetermined by the authors and not taken from the MOJAVE processing. 
Clean I map. Array: BFHKLMNOPS

$2251+158$ at $15.365 \mathrm{GHz} 2008$ Jun 25

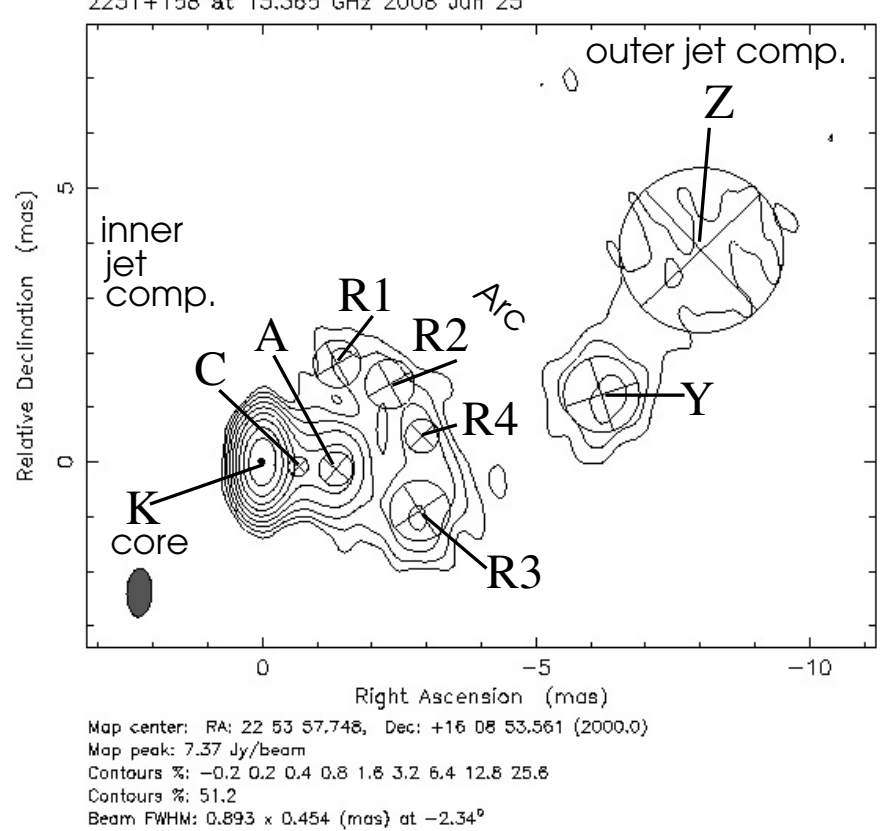

Fig. 1. Image of $2251+158(15 \mathrm{GHz})$ with Gaussian model fit components super-imposed. In addition, the different parts of the parsec-scale morphology are labeled according to their description in the text.

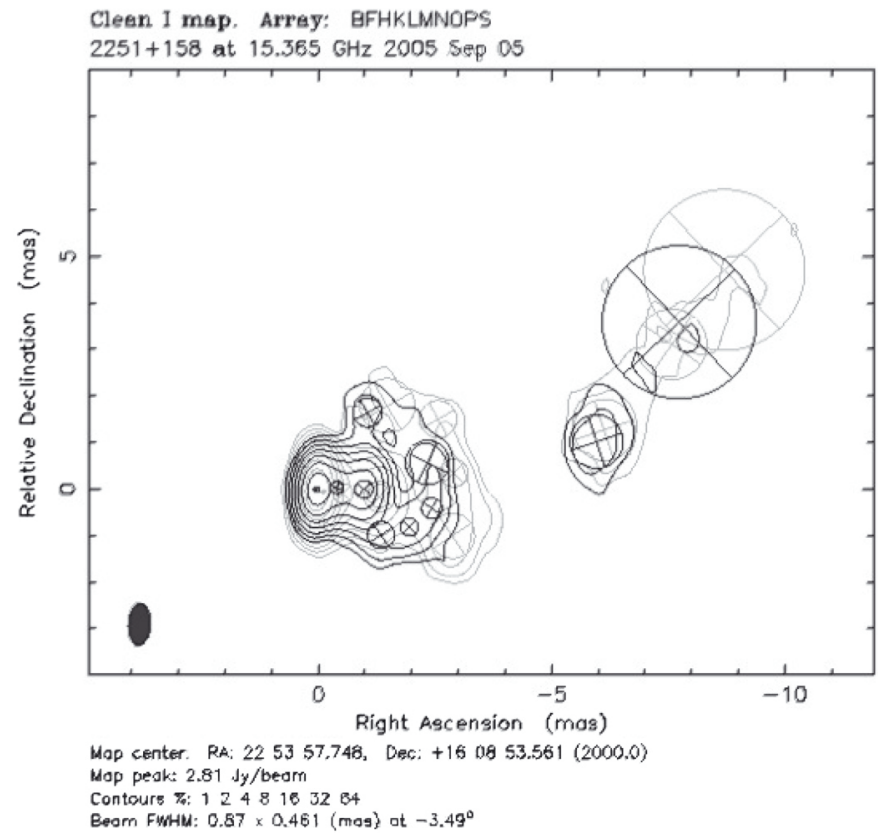

Fig. 2. Two images of $2251+158(15 \mathrm{GHz})$ with Gaussian model fit components superimposed taken on 2005 September 05 (dark black) and on 2008 August 06 (faint). The superposition is meant to visualize the motion of the arc-like structure.

For the following analysis and discussion we assume that the easternmost "feature" of the jet is the core of the AGN, and we refer any apparent velocity calculation to this position. Recent investigations (e.g., Bartel et al. 2012; Ransom et al. 2012) of VLBI observations at $8.4 \mathrm{GHz}$ to estimate the stability of the "core" component of $2251+158$ as reference point for GRAVITY Probe $B$ revealed that the core is stationary within $1 \sigma$ upper limits on its proper motion of $39 \mu$ as $\mathrm{yr}^{-1}$, for the period 2002-2005. We want to stress that for this source the determination of the core position is problematic, as discussed already by Pauliny-Toth et al. (1987).

To determine the individual kinematic properties and apparent motions of these components, we show in Fig. 7 the distance from the core as function of time for all the jet components that have been identified in the jet. The decomposition into substructures and the analysis of their individual properties yield significantly differently evolving parts of the jet. We show them in detail in the following figures. Figure 8 shows the core separation (top), position angle (middle), and flux density (bottom) as a function of time for the inner jet components. Figures 9 and 10 display the equivalent relations for the arc-components and the outer jet components, respectively. In the following sections, we analyze the properties of the substructures in more detail.

\subsection{The presumed core and the inner jet components}

The inner jet region consists of the core $(\mathrm{K})$ and, for the time between 1995 and 2008, the two components A and B. From 2008 and 2010 onward, two additional jet components (C and D, respectively) can be identified in the inner region close to the core. In typical quasars we expect to see apparent superluminal motion for the components in close proximity to the core. However, the situation is different for $2251+158$. Between 1995 and 2005, A and B hardly show any apparent motion away from the core, as can be seen in Fig. 8 (top). However, the position angle of component B changes significantly and steadily from -60 to -105 degrees (Fig. 8, middle). Thus, although components A and B do slowly move away from the core - that is, radially -, they do move with high velocity transverse to the direction of the core. Between 1996.5 and 1999.0, component A moves 0.22 mas, for a speed of 0.087 mas $\mathrm{yr}^{-1}$, or 4.1c. Similarly, component B, between years 1996 and 2003

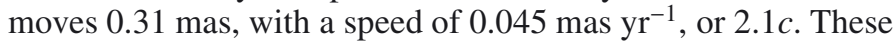
motions are comparable to the radial motions that happen after 2005. When analyzing the kinematics of jet component motion, not only the radial direction should be taken into account (which is usually the case) but the motion in transverse direction as well. We have shown this for several AGN (e.g., 1803+784: Britzen et al. 2010a, 0716+714: Britzen et al. 2009).

Components A and B clearly accelerate and show significantly faster apparent motion (in the radial direction from the core) in the time from about 2005 onwards. Based on the results presented here, we do not find evidence for new component ejections within the 10 year period between 1995 and 2005 .

Around 2005 the two inner components (A and B) start moving away from the core with apparent superluminal speeds of $2.2 \pm 0.5 c$ and $5.5 \pm 0.5 c$, respectively. The new components $C$ and $\mathrm{D}$ move with apparent speeds of $6.4 \pm 0.9 c$ and $7.7 \pm 0.8 c$.

To provide an overview, we list in Table 1 the proper motions, apparent velocities, and back-extrapolated times of ejection from the central region for the different substructures and time periods of observations (via regression analysis). Component A and B show slow apparent radial motion between 1995 and 2005. We do not list the estimated ejection times for this period of time. Examples where the relationship between flaring and component ejection has been questioned for other AGN have been presented by some of the authors (Britzen et al. 2010a,b).

Comparing the parameters we derived for the kinematics and possible ejection times for components $\mathrm{C}$ and $\mathrm{D}$ with those from Jorstad et al. (2012; based on 49 VLBA observations at $43 \mathrm{GHz}$ ), 
A\&A 557, A37 (2013)

Table 1. Parameters of the regression analysis performed for the different components of the jet.

\begin{tabular}{lcccc}
\hline \hline Feature & Time period & $\begin{array}{c}\text { Proper motion } \\
{\left[\mathrm{mas} \mathrm{yr}^{-1}\right]}\end{array}$ & $\begin{array}{c}\beta_{\text {app }} \\
{[c]}\end{array}$ & Est. time of ejection \\
\hline A (inner jet comp.) & $1996.37-2003.24$ & $0.005 \pm 0.011$ & $0.2 \pm 0.5$ & \\
& $2005.68-2011.48$ & $0.046 \pm 0.011$ & $2.2 \pm 0.5$ & $\sim 1995.0$ \\
B (inner jet comp.) & $1996.37-2003.24$ & $0.028 \pm 0.011$ & $1.3 \pm 0.5$ & \\
& $2005.30-2011.28$ & $0.117 \pm 0.011$ & $5.5 \pm 0.5$ & $\sim 1997.4$ \\
C (inner jet comp.) & $2008.48-2011.48$ & $0.137 \pm 0.019$ & $6.4 \pm 0.9$ & $\sim 2007.3$ \\
D (inner jet comp.) & $2010.14-2011.48$ & $0.165 \pm 0.017$ & $7.7 \pm 0.8$ & $\sim 2009.6$ \\
\hline R1 (Arc comp.) & $1996.37-2010.65$ & $0.115 \pm 0.007$ & $5.4 \pm 0.3$ & $\sim 1988.4$ \\
R3 (Arc comp.) & $1995.96-2011.48$ & $0.192 \pm 0.009$ & $9.0 \pm 0.4$ & $\sim 1992.7$ \\
\hline Y (outer jet comp.) & $1995.57-2011.48$ & $0.068 \pm 0.003$ & $3.2 \pm 0.1$ & \\
Z (outer jet comp.) & $1995.57-2011.48$ & $0.066 \pm 0.010$ & $3.1 \pm 0.5$ & \\
\hline
\end{tabular}

it seems likely that component $\mathrm{C}$ in this paper corresponds to component $\mathrm{K} 1$ in Jorstad et al. Component D (this paper) could be identical with component K09 (Jorstad et al.). Taking into account possible frequency dependant position shifts and the uncertainties in the estimation of the ejection times, these crossidentifications seem secure.

We want to mention the flux density variability of the core (Fig. 8, bottom) and the high flux density value for D, which exceeds by far that of the core around 2010 - the time of the major multi-wavelength flare. The total flux density in the radio at that time as measured within the University of Michigan Radio Astronomy Observatory (UMRAO)-monitoring program reaches values above $28 \mathrm{Jy}$ at $14.5 \mathrm{GHz}$. The core has an average value of around $6 \mathrm{Jy}$ and D's flux density rises up to $18 \mathrm{Jy}$.

Summarizing the findings: we observe two different kinematic modes for component motion in this source. Until 2005 two components remain almost stationary with regard to the core and no component ejections are observed in the data presented here. These two components however do show very high velocities transverse to the direction of the core. From 2005 onward, the components move with apparent superluminal speeds away from the core and two new components seem to be ejected. The transition coincides with the start of major broad-band flaring activity which is reported and discussed in detail in several publications (e.g., Ogile et al. 2011; Vol'vach et al. 2011b).

\subsection{The arc-like feature}

We detect an arc-like structure around the parsec-scale core in maps at $15 \mathrm{GHz}$ and at $43 \mathrm{GHz}$, see Figs. 3-6. The arc is visible in the images at both frequencies. At $15 \mathrm{GHz}$ the morphology can be described as roughly a quarter of a ring and is located towards the northwest of the core. Based on our $43 \mathrm{GHz}$ maps it seems that the $43 \mathrm{GHz}$ arc extends further towards the south compared to the $15 \mathrm{GHz}$ data. This structure developed significantly over the time of the observations. To our knowledge, this is the first source for which such a kind of arc is reported. First signs of the arc-phenomenon can be seen in Fig. 3i.

Since this kind of feature is a novelty compared to other more typical structures, we tested several scenarios for the representation and identification of this morphology. We report the scenario that represents the phenomenon best: the decomposition into a fixed number of discrete components. We are confident that we could trace these components across all epochs, but we allowed in the fitting process that not all the components are visible in all the epochs. While this seems to be the most robust and straight-forward way of representing the structure currently, we do not rule out that once the physical nature of this feature is solved, a different way of representation might even be more adequate.

We fit five Gaussian components (R0-R4) to the model-fit data. The results are shown in Fig. 9 for the four best-traced arcfeatures. The plot at the top shows the core separation versus time for those four components that could be traced most reliably through time. All components move away from the core. To measure the apparent velocity of this expansion, we calculated linear regression models to the core separations as function of time for R1 to R4 (Fig. 9, top). We list the values for the two besttraced arc-features in Table 1 . The estimated velocities range be-

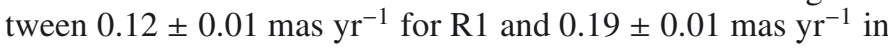
the case of R3. Based on the back-extrapolation of the observed expansion, the ejection of this arc could have been generated in the core region between 1988 and 1992. To check for the direction of the motion of the arc, we show the position angle evolution of the four arc-components in Fig. 9 (middle). The motion can be more clearly seen in Fig. 11 in Cartesian coordinates. While the paths of R1, R2, and R3 seem to describe bows, the path of R4 is best represented by a straight line. Fig. 9 (bottom) displays the flux density evolution of R1 to R4, with R3 showing a significant rise in flux density in 2006.

The arc-like structure is expanding, i.e., increasing in diameter with time. This can be seen in Fig. 2 where we superimposed two images of the source taken three years apart in time. At the time of the submission of this paper the arc-like feature was still definitely present, but it obviously had become fainter.

\subsection{The outer jet components}

All of the trajectories of the knots and associated plasma flows converge to reach a narrow area around the position of $(-3$ mas, -1 mas). After this point the track of the jet plasma flow changes its direction sharply. Beyond the position ( -3 mas, -1 mas), the jet flow is characterized by two knots, $\mathrm{Y}$ and $\mathrm{Z}$. Figure 10 shows the behavior of jet components at larger core distances, where the jet appears to be well-collimated. Both components $\mathrm{Y}$ and $\mathrm{Z}$ separate from the core with proper mo-

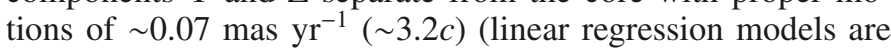
superimposed in the core separation versus time plot in Fig. 10, top). These components were known from earlier observations. 
The position angles of their trajectories are stable (see Fig. 10, middle). Relatively stable as well are the flux densities of these outer features (Fig. 10, bottom). The outer jet components show the typical behavior expected in AGN jets.

\section{Flux density evolution}

The radio flux density of $2251+158$ is known to be highly variable across the electromagnetic spectrum. In the following, we discuss whether the variability observable in the radio regime shows correlations with the morphological changes as seen in the VLBI observations.

In Fig. 12 (top) we show the single-dish radio flux densities as monitored within the UMRAO monitoring program (4.8, 8.0, and $14.5 \mathrm{GHz})$. Optical $R$-band data monitored with the Abastumani telescope are superimposed. Figure 12 (bottom) shows an expansion of the part of the light-curve which shows radio as well as optical information.

The most prominent long-term radio-variability seems to occur simultaneously at the three frequencies. The radio lightcurves since the late 1960s reveal a quite characteristic signature of three major flares followed by a couple of smaller flares. The number of smaller flares increased after the major flare recorded in 1995. The last dominant radio flare in 2010 was part of a significant multi-wavelength event monitored by many telescopes (e.g., Vol'vach et al., 2011a,b).

Qian et al. (2007) modeled the characteristic flux density variability pattern at 4.8 and $8 \mathrm{GHz}$ with a binary black hole model.

The optical data clearly show strong variability. A detailed correlation analysis is required and will be published in a forthcoming paper.

We find the first hint for an arc in VLBI maps at $15 \mathrm{GHz}$ around 1996. From this time onwards the arc expands and becomes more clearly visible. We discuss the possible physical nature of the arc in the discussion (Sect. 5). If the arc is related to material that was ejected from the central region, it is likely that this happened before the major flare around 1995, probably during the minimum.

When the arc is visible in the VLBI maps and model-fits, $2251+158$ undergoes dramatic flux density flaring across the spectrum from the radio to the high energies. This broad-band variability is observed while the arc is already part of the VLBIstructure on parsec scales.

\section{Discussion}

We find a complex set of morphological and kinematical phenomena in and around the core region of this quasar. While these structures are robust in data analysis and present significant physical structures, it is not easily possible to put the pieces together and explain the apparent complexity with one physical scenario. We summarize the findings before we discuss probable physical models:

- An arc-like feature appears around the core region of $2251+158$.

- The arc expands with an apparent superluminal speed between $5.4 \pm 0.3 c$ and $9.0 \pm 0.4 c$ until 2010 .

- The arc fades after 2010.

- The jet is well-collimated from about 3 mas core distance onwards.
- The inner jet components show slow apparent radial motion between 1995 and 2005 (slow apparent motion had been reported earlier by Pauliny-Toth et al. 1987).

- No component ejections are observed between 1995 and 2005 (a phase without component ejections had been reported earlier by Pauliny-Toth et al. 1987).

- The inner jet components start moving with apparent superluminal speeds after 2005.

- Component ejections are observed after 2005.

- Component B shows a steady decline in position angle for about 15 years.

- The light-curve variability might be correlated with the observed morphological features (arc, kinematics of inner jet components, ejection of components).

\subsection{Peculiar kinematics}

Based on the observations and findings reported by Pauliny-Toth et al. (1984, 1987), after a major radio flare in 1981 the components close to the core remained at similar core distances for some time - not showing the typical apparent superluminal motion expected for this kind of object and thus appearing to be stationary.

These structural changes, as reported by Pauliny-Toth et al., do not appear to fit easily into the scheme of relativistic jet models. The structural variations the authors report appear to be much more complex ("superluminal brightening"). The observations show a rapid increase in size of the core region and the appearance of some features which are nearly stationary, while others move with apparent velocities of $20 c$. Pauliny-Toth et al. conclude that the most likely scenario to explain their observations is that the features in the core region may not represent discrete, physical components, but rather regions where the emission from a relativistic jet is enhanced, for example by shocks. Pauliny-Toth et al. refer to Lind \& Blandford (1985), who have emphasized that the velocity of shock fronts, which might be observed as a motion of "components", can be smaller than that of the underlying medium, which determines the Doppler boosting of the emission. In particular, it seems possible to obtain stationary emission patterns. Near the core, the shock pattern appears stationary. A change in physical conditions at larger distances from the core produces a moving pattern and gives rise to the "classical" superluminal behavior of the outer components.

We find similar slow apparent-motion for the two inner components for the time after the major radio flare in 1995. However, since high apparent velocities are observed transverse to the direction of the core in the data discussed in this paper, this phenomenon seems to be better described as apparent radial stationarity. Between 1995 and 2005 no component ejections are seen. The kinematic behavior appears to change for the inner two components in 2005. From 2005 onwards all four components move with apparent superluminal speeds. Thus, the apparent speeds of the jet components most likely changed around $2005-$ assuming that these are the same components. Not only did the kinematic properties of the jet components change - the variability properties of the light-curve also changed (see e.g., Vol'vach et al. 2011b). While the kinematic properties, such as the apparent speed usually remain similar in a given source over many years of observations, we find in the case of $2251+158$ that the kinematic properties can change with time. Phases of apparent slow radial motion can alternate with phases of apparent fast (superluminal) speeds. While the creation and appearance of the arc could be a one-time, one-source phenomenon, 
the jet ridge line and kinematic changes have been reported and investigated by us in some other AGN as well. Kinematic mode changes have been reported in the case of the AGN 0735+178 (Britzen et al. 2010b). We reported three mode changes in the kinematic properties (from stationary to apparent superluminal motion) within 30 years. These mode changes correlate with significant changes of the morphology and correlate as well with flaring observed in the optical light-curve.

While $0735+178$ has been classified as a BL Lac object, with a relativistic jet oriented close to the line of sight, 2251+158 is classified as quasar. Within radio-loud unification schemes (e.g., Antonucci 1993; Urry \& Padovani 1995) quasars belong to the high-luminosity class of AGN, while the BL Lac objects tend to be less luminous objects. $2251+158$ is the first quasar where we observed a change in kinematic properties - similar to the mode changes observed in the BL Lac object $0735+178$. The detectability of variations in the kinematic properties requires long-term VLBI monitoring and going back to archival data or early publications of VLBI observations. The latter might have been obtained with different VLBI arrays or at different observing frequencies. While this can cause some problems comparing observations of different quality, frequency, or dynamic range, in the cases of both $0735+178$ and $2251+158$, the effect seems to be significant. Searching for such kinematic changes in other AGN, where this long-term VLBI monitoring is available, might yield more sources showing this behaviour.

\subsection{Possible explanations for the observed phenomena}

Unfortunately, not all of the observed features can be explained within one theoretical model.

In particular, the formation of the expanding arc structure is a challenge. Another challenge is how to explain that all the trajectories of the knots and associated plasma flows converge to reach a narrow area around the position of ( -3 mas, -1 mas). After this point the track of the jet plasma flow changes its direction sharply. Assuming that the arc-like feature is part of the jet or a shock, it is difficult to explain how the jet which became broader (arc) can be collimated again at the position of ( -3 mas, -1 mas).

In the following we mention briefly some physical scenarios that could explain at least part of the observed phenomena. Detailed analysis and modeling testing the proposed scenarios is in preparation and the results will be published in forthcoming papers.

Currently we can present and discuss several alternative explanations but not claim to have one model capable of explaining all the observed phenomena.

\subsubsection{Apparent superluminal motion and apparent radially stationary jet components}

Traditionally, due to lower resolution images, the outward directed motion of jet components has been studied and the absence of high apparent speeds in the radial direction (towards or away from the core) has been named "stationary". The results of some theoretical studies and simulations of relativistic jets (e.g., Lind \& Blandford 1985; Daly \& Marscher 1988; Gómez et al. 1997; Agudo et al. 2001) may be applied to explain the coexistence of these stationary features and the classical apparent superluminal motion.

\subsubsection{Possible explanation for the convergence of the plasma flow}

The convergence of the plasma flow could be caused by an inhomogeneous medium distribution surrounding the core. Beyond the position ( -3 mas, -1 mas) the jet flow is characterized by two knots $\mathrm{Y}$ and $\mathrm{Z}$. These two knots could be produced by plasma blobs moving through standing shocks, or by compression by an increasing field strength and electron density. This has been modeled successfully by Daly \& Marscher (1988).

\subsubsection{Possible origin of the arc-feature}

The feature could have evolved from an arc structure initially ejected from the core. It could also be caused by a helical jet (or magnetic field) and shock propagation along the jet. Extended jet regions could become visible due to such shock propagation.

As a possible alternative model, a precessing jet could explain the observed apparent stationarity with regard to the radial component of the observed motion (for at least ten years) of the inner jet components, the arc due to projection effects and possible relativistic amplification, as well as the observed variability patterns across the wavelength spectrum. The jet would point in one direction for some time and would then appear differently when pointing in another direction. In this case the relativistic amplification would change and so would the apparent speeds. This model is supported by the changing jet structure as seen in the $15 \mathrm{GHz}$ maps, and the trajectories of the apparent superluminal knots. The precession scenario is supported by the observations of Pauliny-Toth et al. (1998) - suggesting that a similar jet component motion had already been observed some time ago.

Thus, the complex phenomena observed in $2251+158$ could result from a relativistic jet flow pattern in an exceptional environment: a precessing jet and its interaction with the interstellar medium surrounding the core, involving the formation of shocks and shear-layers. By monitoring the VLBI and lightcurve properties of this source it should be possible to determine the timescale of the precession for this model. The precession could originate in a supermassive binary black hole system. A theoretical model for fitting the kinematics of the superluminal knots in terms of jet-precession is in preparation (Qian et al.).

Acknowledgements. Special thanks go to J. Anderson for carefully reading the manuscript and providing valuable comments to improve the manuscript. We thank J. Attridge, J.-L. Gómez and A. Marscher for providing VLBI data of $2251+158$ from their AGN monitoring programs. Part of this work was supported by the COST Action MP0905 Black Holes in a Violent Universe. We appreciate the use of the NRAO archive of VLBA data. This research has made use of data from the MOJAVE database that is maintained by the MOJAVE team (Lister et al. 2009). This work made use of the VLBA, which is a facility of the National Science Foundation, operated under cooperative agreement by Associated Universities, Inc. This research has made use of data from the University of Michigan Radio Astronomy Observatory which is supported by the National Science Foundation and by funds from the University of Michigan. This research has made use of the NASA/IPAC Extragalactic Database (NED) which is operated by the Jet Propulsion Laboratory, California Institute of Technology, under contract with the National Aeronautics and Space Administration. The National Radio Astronomy Observatory is a facility of the National Science Foundation operated under cooperative agreement by Associated Universities, Inc. This work is partly supported by the Georgian National Science Foundation through grant GNSF/ST09-521-4-320.

\section{References}

Ackermann, M., Ajello, M., Baldini, L., et al. 2010, ApJ, 721, 1383 Agudo, I., Gómez, J.-L., Martí, J.-M., et al. 2001, ApJ, 549, L183 
Antonucci, R. 1993, ARA\&A, 31, 473

Bartel, N., Bietenholz, M. F., Lebache, D. E., et al. 2012, ApJS, 201, 7 Bonnoli, G., Ghisellini, G., Foschini, L., et al. 2011, MNRAS, 410, 368 Britzen, S., Vermeulen, R. C., Campbell, R. M., et al. 2008, A\&A, 484, 119 Britzen, S., Kam, V. A., Witzel, A., et al. 2009, A\&A, 508, 1205

Britzen, S., Kudryavtseva, N. A., Witzel, A., et al. 2010a, A\&A, 511, A57

Britzen, S., Witzel, A., Gong, B. P., et al. 2010b, A\&A, 515, A105

Daly, R., \& Marscher, A. P. 1988, ApJ, 334, 539

Fomalont, E. B., Frey, S., Paragi, Z., et al. 2000, ApJS, 131, 95

Gómez, J.-L., Marti, J. M. A., Marscher, A. P., et al. 1997, ApJ, 482, L33

Gong, B. 2008, MNRAS, 389, 315

Homan, D. C., \& Lister, M. L. 2006, AJ, 131, 1262

Jorstad, S. G., Marscher, A. P., Larionov, V. M., et al. 2010, ApJ, 715, 362

Jorstad, S. G., Marscher, A. P., Joshi, M., et al. 2012, Fermi \& Jansky

Proceedings [arXiv: 1205.0520 ]

Kellermann, K. I., Lister, M. L., Homan, D. C., et al. 2004, ApJ, 609, 539

Kemball, A. J., Diamond, P. J., \& Pauliny-Toth, I. I. K. 1996, ApJ, 464, 55

Lind, K. R., \& Blandford, R. D. 1985, ApJ, 295, 358

Lister, M. L., \& Homan, D. C. 2005, AJ, 130, 1389

Lister, M. L., Cohen, M. H., Homan, D. C., et al. 2009, AJ, 138, 1874

Lobanov, A. P., Krichbaum, T. P., Graham, D. A., et al. 2000, A\&A, 364, 391
Lonsdale, C. J., Doeleman, S., \& Phillips, R. B. 1998, AJ, 16, 8

Marscher, A. P., Jorstad, S. G., Mattox, J. R., et al. 2002, ApJ, 577, 85

Moellenbrock, G. A., Fujisawa, K., Preston, R. A., et al. 1996, AJ, 111, 217

Pauliny-Toth, I. I. K. 1998, ASP, 144, 75

Pauliny-Toth, I. I. K., Porcas, R. W., Zensus, J. A., \& Kellermann, K. I. 1984 (Dordrecht: Reidel), IAU Symp., 110, 149

Pauliny-Toth, I. I. K., Porcas, R. W., Zensus, J. A., et al. 1987, Nature, 328, 778

Qian, S.-J., Kudryavtseva, N. A., Britzen, S., et al. 2007, ChJAA, 7, 364

Raiteri, C. M., Villata, M., Aller, M. F., Gurwell, M. A., et al. 2011, A\&A, 534, A87

Ransom, R. R., Bartel, N., Bietenholz, M. F., et al. 2012, ApJS, 201, 2

Rantakyro, F. T., Baath, L. B., Backer, et al. 1998, A\&AS, 131, 451

Savolainen, T., Wiik, K., Valtaoja, E., et al. 2002, A\&A, 394, 851

Tosti, G., Chiang, J., Lott, B., et al. 2008, ATel, 1634

Urry, P., \& Padovani, P. 1995, PASP, 107, 803

Vercellone, S. 2012, Fermi \& Jansky Proceedings [arXiv: 1205.5510]

Vol'vach, A. E., Vol'vach, L. N., Kut'kin, A. M., et al. 2011a, Astron. Rep., 55, 608

Vol'vach, A. E., Kut'kin, A. M., Vol'vach, L. N., et al. 2011b, Astrophysics, 54, 363

Wehrle, A. E., Marscher, A. P., Jorstad, S. G., et al. 2012, ApJ, 758, 72

Pages 8 to 17 are available in the electronic edition of the journal at http://www . aanda.org 
Table 2. Model-fit parameters for $2251+158$

\begin{tabular}{|c|c|c|c|c|c|}
\hline $\begin{array}{l}\text { Epoch } \\
\text { (1) }\end{array}$ & $\begin{array}{c}\text { FD } \\
{[\mathrm{Jy}]} \\
(2)\end{array}$ & $\begin{array}{c}\text { C Sep } \\
\text { [mas] } \\
(3)\end{array}$ & $\begin{array}{c}\text { PA } \\
\text { [deg] } \\
(4)\end{array}$ & $\begin{array}{c}\mathrm{MA} \\
{[\mathrm{mas}]} \\
(5)\end{array}$ & $\begin{array}{l}\text { Id } \\
\text { (6) }\end{array}$ \\
\hline 1995.57 & 5.274 & 0.00 & 0.0 & 0.21 & $\mathrm{~K}$ \\
\hline 1995.57 & 1.367 & 0.51 & -96.6 & 0.11 & $\mathrm{x}$ \\
\hline 1995.57 & 3.744 & 0.54 & -57.5 & 0.29 & $\mathrm{x}$ \\
\hline 1995.57 & 0.576 & 0.92 & -109.5 & 0.69 & $\mathrm{x}$ \\
\hline 1995.57 & 0.046 & 2.12 & -101.1 & 0.49 & $\mathrm{x}$ \\
\hline 1995.57 & 0.601 & 5.22 & -86.8 & 2.79 & Y \\
\hline 1995.57 & 0.593 & 8.40 & -63.5 & 3.49 & $\mathrm{Z}$ \\
\hline 1995.96 & 2.132 & 0.00 & 0.0 & 0.17 & K \\
\hline 1995.96 & 3.000 & 0.41 & -68.5 & 0.25 & $\mathrm{x}$ \\
\hline 1995.96 & 0.692 & 0.66 & -51.7 & 0.22 & $\mathrm{x}$ \\
\hline 1995.96 & 0.322 & 0.78 & -113.1 & 0.34 & R3 \\
\hline 1995.96 & 0.182 & 5.24 & -86.3 & 0.94 & $\mathrm{Y}$ \\
\hline 1995.96 & 0.437 & 8.40 & -63.5 & 4.95 & $\mathrm{Z}$ \\
\hline 1996.37 & 2.462 & 0.00 & 0.0 & 0.17 & K \\
\hline 1996.37 & 5.826 & 0.44 & -65.1 & 0.23 & A \\
\hline 1996.37 & 0.865 & 0.70 & -103.4 & 0.90 & B \\
\hline 1996.37 & 0.395 & 0.81 & -38.6 & 0.01 & $\mathrm{R} 1$ \\
\hline 1996.37 & 0.181 & 1.16 & -37.2 & 0.70 & $\mathrm{x}$ \\
\hline 1996.37 & 0.040 & 2.50 & -117.6 & 0.22 & $\mathrm{x}$ \\
\hline 1996.37 & 0.494 & 5.46 & -84.4 & 1.23 & Y \\
\hline 1998.18 & 1.134 & 0.00 & 0.0 & 0.13 & K \\
\hline 1998.18 & 3.530 & 0.52 & -68.6 & 0.24 & A \\
\hline 1998.18 & 0.956 & 0.62 & -93.8 & 0.20 & B \\
\hline 1998.18 & 0.120 & 1.07 & -107.4 & 0.25 & R3 \\
\hline 1998.18 & 0.133 & 1.29 & -29.2 & 0.29 & $\mathrm{R} 1$ \\
\hline 1998.18 & 0.131 & 1.58 & -58.9 & 0.90 & $\mathrm{R} 2$ \\
\hline 1998.18 & 0.037 & 2.04 & -93.0 & 0.30 & $\mathrm{x}$ \\
\hline 1998.18 & 0.089 & 3.99 & -109.9 & 2.01 & $\mathrm{x}$ \\
\hline 1998.18 & 0.431 & 5.77 & -83.6 & 1.33 & Y \\
\hline 1998.18 & 0.362 & 8.08 & -63.7 & 3.52 & $\mathrm{Z}$ \\
\hline 1998.83 & 1.418 & 0.00 & 0.0 & 0.15 & K \\
\hline 1998.83 & 4.312 & 0.53 & -72.3 & 0.48 & A \\
\hline 1998.83 & 1.747 & 0.73 & -80.7 & 0.01 & B \\
\hline 1998.83 & 0.332 & 0.81 & -109.3 & 0.37 & R0 \\
\hline 1998.83 & 0.140 & 1.21 & -30.4 & 0.24 & $\mathrm{R} 1$ \\
\hline 1998.83 & 0.132 & 1.55 & -109.7 & 0.85 & R3 \\
\hline 1998.83 & 0.037 & 2.01 & -66.2 & 0.40 & $\mathrm{R} 2$ \\
\hline 1998.83 & 0.462 & 5.81 & 81.5 & 1.92 & $\mathrm{Y}$ \\
\hline 1998.83 & 0.239 & 8.33 & -66.1 & 2.21 & $\mathrm{Z}$ \\
\hline 1998.83 & 0.098 & 10.65 & -57.9 & 1.83 & $\mathrm{w}$ \\
\hline 1999.55 & 1.108 & 0.00 & 0.0 & 0.13 & K \\
\hline 1999.55 & 4.865 & 0.59 & -73.2 & 0.21 & A \\
\hline 1999.55 & 2.061 & 0.69 & -91.6 & 0.44 & B \\
\hline 1999.55 & 0.597 & 0.87 & -36.6 & 0.38 & $\mathrm{x}$ \\
\hline 1999.55 & 0.202 & 1.48 & -109.3 & 0.79 & R3 \\
\hline 1999.55 & 0.095 & 1.52 & -45.5 & 0.74 & $\mathrm{R} 1$ \\
\hline 1999.55 & 0.073 & 3.07 & -101.6 & 1.27 & $\mathrm{x}$ \\
\hline 1999.55 & 0.357 & 5.77 & -83.5 & 0.94 & Y \\
\hline 1999.55 & 0.605 & 8.00 & -66.8 & 5.05 & $\mathrm{Z}$ \\
\hline 2000.49 & 4.697 & 0.00 & 0.0 & 0.19 & K \\
\hline 2000.49 & 1.290 & 0.55 & -74.2 & 0.33 & A \\
\hline 2000.49 & 0.228 & 0.69 & -25.7 & 0.19 & $\mathrm{x}$ \\
\hline 2000.49 & 1.042 & 0.72 & -101.0 & 0.41 & B \\
\hline 2000.49 & 0.438 & 1.01 & -90.6 & 0.79 & $\mathrm{R} 4$ \\
\hline 2000.49 & 0.533 & 1.06 & -29.3 & 0.65 & $\mathrm{x}$ \\
\hline 2000.49 & 0.170 & 2.25 & -100.6 & 2.16 & $\mathrm{x}$ \\
\hline
\end{tabular}

Notes. Column (1) gives the epoch of observation, Col. (2) gives the integrated flux density of an individual component, Col. (3) gives the core separation, Col. (4) gives the position angle from the core, Col. (5) gives the angular size of the component, and Col. (6) gives the identifier of the component.
Table 2. continued.

\begin{tabular}{|c|c|c|c|c|c|}
\hline Epoch & $\begin{array}{l}\text { FD } \\
{[\mathrm{Jy}]} \\
(2)\end{array}$ & $\begin{array}{c}\text { C Sep } \\
{[\mathrm{mas}]} \\
(3)\end{array}$ & $\begin{array}{c}\text { PA } \\
{[\mathrm{deg}]} \\
(4)\end{array}$ & $\begin{array}{c}\text { MA } \\
{[\text { mas }]} \\
\text { (5) }\end{array}$ & Id \\
\hline 2000.49 & 0.449 & 5.68 & -81.6 & 1.14 & $\mathrm{Y}$ \\
\hline 2000.49 & 0.462 & 8.18 & -67.1 & 4.17 & $\mathrm{Z}$ \\
\hline 2001.06 & 1.477 & 0.00 & 0.0 & 0.27 & $\mathrm{~K}$ \\
\hline 2001.06 & 3.503 & 0.45 & -79.8 & 0.20 & A \\
\hline 2001.06 & 0.910 & 0.84 & -103.8 & 0.59 & B \\
\hline 2001.06 & 0.335 & 1.02 & -22.8 & 0.39 & $\mathrm{x}$ \\
\hline 2001.06 & 0.212 & 1.30 & -81.7 & 0.83 & R4 \\
\hline 2001.06 & 0.163 & 1.43 & -33.7 & 0.58 & $\mathrm{R} 1$ \\
\hline 2001.06 & 0.101 & 3.45 & -110.4 & 1.82 & $\mathrm{x}$ \\
\hline 2001.06 & 0.334 & 5.77 & -81.6 & 0.96 & $\mathrm{Y}$ \\
\hline 2001.06 & 0.388 & 8.06 & -66.7 & 4.26 & $\mathrm{Z}$ \\
\hline 2003.24 & 3.102 & 0.00 & 0.0 & 0.16 & K \\
\hline 2003.24 & 1.416 & 0.51 & -85.9 & 0.50 & A \\
\hline 2003.24 & 2.934 & 0.84 & -91.1 & 0.28 & B \\
\hline 2003.24 & 0.042 & 0.85 & 81.9 & 0.01 & \\
\hline 2003.24 & 0.430 & 1.32 & -119.5 & 0.56 & R0 \\
\hline 2003.24 & 0.299 & 1.62 & -33.3 & 0.57 & $\mathrm{R} 1$ \\
\hline 2003.24 & 0.238 & 1.99 & -81.7 & 0.66 & R4 \\
\hline 2003.24 & 0.115 & 1.99 & -112.5 & 0.33 & R3 \\
\hline 2003.24 & 0.066 & 3.51 & -103.5 & 1.09 & $\mathrm{x}$ \\
\hline 2003.24 & 0.386 & 5.94 & -80.2 & 1.19 & $\mathrm{Y}$ \\
\hline 2003.24 & 0.419 & 8.13 & -66.1 & 2.72 & $\mathrm{Z}$ \\
\hline 2003.24 & 0.082 & 10.78 & -60.7 & 1.75 & \\
\hline 2005.30 & 3.041 & 0.00 & 0.0 & 0.31 & K \\
\hline 2005.30 & 0.664 & 0.71 & -97.7 & 0.01 & B \\
\hline 2005.30 & 2.194 & 0.77 & -86.7 & 0.66 & B \\
\hline 2005.30 & 0.123 & 1.77 & -28.2 & 0.37 & R1 \\
\hline 2005.30 & 0.236 & 1.77 & -124.2 & 0.54 & R0 \\
\hline 2005.30 & 0.338 & 2.10 & -102.1 & 0.53 & $\mathrm{R} 3$ \\
\hline 2005.30 & 0.172 & 2.45 & -71.8 & 0.75 & $\mathrm{R} 2$ \\
\hline 2005.30 & 0.401 & 5.92 & -79.6 & 1.31 & $\mathrm{Y}$ \\
\hline 2005.30 & 0.545 & 8.57 & -64.2 & 3.34 & $\mathrm{Z}$ \\
\hline 2005.68 & 2.722 & 0.00 & 0.0 & 0.12 & $\mathrm{~K}$ \\
\hline 2005.68 & 1.352 & 0.44 & -85.8 & 0.28 & A \\
\hline 2005.68 & 2.195 & 1.03 & -89.9 & 0.39 & B \\
\hline 2005.68 & 0.212 & 1.70 & -125.3 & 0.57 & R0 \\
\hline 2005.68 & 0.166 & 1.98 & -33.7 & 0.62 & R1 \\
\hline 2005.68 & 0.236 & 2.15 & -112.1 & 0.39 & R3 \\
\hline 2005.68 & 0.282 & 2.45 & -76.4 & 0.95 & R2 \\
\hline 2005.68 & 0.207 & 2.51 & -99.3 & 0.45 & R4 \\
\hline 2005.68 & 0.390 & 6.09 & -80.3 & 1.15 & $\mathrm{Y}$ \\
\hline 2005.68 & 0.605 & 8.57 & -65.2 & 3.30 & $\mathrm{Z}$ \\
\hline 2005.71 & 2.959 & 0.00 & 0.0 & 0.11 & K \\
\hline 2005.71 & 1.472 & 0.47 & -88.4 & 0.35 & A \\
\hline 2005.71 & 1.994 & 1.04 & -90.7 & 0.37 & B \\
\hline 2005.71 & 0.281 & 2.00 & -42.6 & 1.18 & R1 \\
\hline 2005.71 & 0.710 & 2.06 & -111.5 & 1.10 & R3 \\
\hline 2005.71 & 0.204 & 2.56 & -85.4 & 0.48 & R4 \\
\hline 2005.71 & 0.394 & 6.10 & -80.3 & 1.19 & $\mathrm{Y}$ \\
\hline 2005.71 & 0.561 & 8.62 & -64.7 & 3.20 & $\mathrm{Z}$ \\
\hline 2006.19 & 7.872 & 0.00 & 0.0 & 0.12 & $\mathrm{~K}$ \\
\hline 2006.19 & 1.393 & 0.62 & -87.1 & 0.25 & A \\
\hline 2006.19 & 1.466 & 1.11 & -92.2 & 0.44 & B \\
\hline 2006.19 & 0.236 & 2.01 & -43.1 & 1.19 & $\mathrm{R} 1$ \\
\hline 2006.19 & 0.505 & 2.29 & -109.5 & 0.97 & R3 \\
\hline 2006.19 & 0.210 & 2.60 & -83.4 & 0.58 & R4 \\
\hline 2006.19 & 0.374 & 6.09 & -80.1 & 1.16 & $\mathrm{Y}$ \\
\hline 2006.19 & 0.514 & 8.58 & -64.9 & 3.11 & $\mathrm{Z}$ \\
\hline 2006.45 & 4.154 & 0.00 & 0.0 & 0.13 & K \\
\hline 2006.45 & 1.331 & 0.66 & -91.5 & 0.31 & A \\
\hline 2006.45 & 1.140 & 1.15 & -92.7 & 0.43 & $\mathrm{~B}$ \\
\hline
\end{tabular}


Table 2. continued.

\begin{tabular}{|c|c|c|c|c|c|}
\hline Epoch & $\begin{array}{c}\text { FD } \\
{[\mathrm{Jy}]} \\
(2)\end{array}$ & $\begin{array}{c}\text { C Sep } \\
\text { [mas] } \\
\text { (3) }\end{array}$ & $\begin{array}{c}\text { PA } \\
\text { [deg] } \\
\text { (4) }\end{array}$ & $\begin{array}{c}\text { MA } \\
{[\text { mas] }} \\
(5)\end{array}$ & Id \\
\hline 2006.45 & 0.157 & 1.78 & -32.3 & 0.75 & $\mathrm{R} 1$ \\
\hline 2006.45 & 0.449 & 2.37 & -109.6 & 0.82 & R3 \\
\hline 2006.45 & 0.091 & 2.51 & -58.0 & 0.83 & R2 \\
\hline 2006.45 & 0.196 & 2.62 & -84.7 & 0.46 & $\mathrm{R} 4$ \\
\hline 2006.45 & 0.047 & 3.78 & -102.6 & 0.51 & $\mathrm{x}$ \\
\hline 2006.45 & 0.361 & 6.09 & -79.7 & 1.06 & Y \\
\hline 2006.45 & 0.551 & 8.52 & -64.0 & 3.45 & $\mathrm{Z}$ \\
\hline 2007.61 & 2.710 & 0.00 & 0.0 & 0.13 & $\mathrm{~K}$ \\
\hline 2007.61 & 0.552 & 0.40 & -97.1 & 0.41 & A \\
\hline 2007.61 & 1.441 & 1.17 & -94.6 & 0.53 & B \\
\hline 2007.61 & 0.084 & 2.13 & -32.8 & 0.85 & $\mathrm{R} 1$ \\
\hline 2007.61 & 0.128 & 2.65 & -56.8 & 0.93 & R2 \\
\hline 2007.61 & 0.485 & 2.75 & -107.8 & 1.07 & R3 \\
\hline 2007.61 & 0.226 & 2.79 & -81.6 & 0.56 & $\mathrm{R} 4$ \\
\hline 2007.61 & 0.412 & 6.24 & -79.1 & 1.26 & $\mathrm{Y}$ \\
\hline 2007.61 & 0.489 & 8.81 & -64.7 & 2.84 & $\mathrm{Z}$ \\
\hline 2008.48 & 6.929 & 0.00 & 0.0 & 0.10 & K \\
\hline 2008.48 & 1.095 & 0.24 & -107.2 & 0.00 & $\mathrm{C}$ \\
\hline 2008.48 & 0.323 & 0.68 & -97.6 & 0.36 & A \\
\hline 2008.48 & 0.780 & 1.37 & -95.6 & 0.62 & B \\
\hline 2008.48 & 0.092 & 2.26 & -37.8 & 0.87 & R1 \\
\hline 2008.48 & 0.120 & 2.74 & -58.9 & 0.90 & $\mathrm{R} 2$ \\
\hline 2008.48 & 0.172 & 2.97 & -80.9 & 0.62 & $\mathrm{R} 4$ \\
\hline 2008.48 & 0.485 & 3.03 & -107.1 & 1.11 & R3 \\
\hline 2008.48 & 0.414 & 6.34 & -78.8 & 1.39 & $\mathrm{Y}$ \\
\hline 2008.48 & 0.504 & 8.92 & -64.3 & 3.01 & $\mathrm{Z}$ \\
\hline 2008.55 & 7.290 & 0.00 & 0.0 & 0.05 & K \\
\hline 2008.55 & 1.780 & 0.20 & -104.8 & 0.01 & $\mathrm{C}$ \\
\hline 2008.55 & 0.453 & 0.62 & -95.8 & 0.46 & A \\
\hline 2008.55 & 0.728 & 1.39 & -96.2 & 0.63 & B \\
\hline 2008.55 & 0.077 & 2.11 & -37.1 & 0.96 & R1 \\
\hline 2008.55 & 0.149 & 2.73 & -57.7 & 1.05 & $\mathrm{R} 2$ \\
\hline 2008.55 & 0.161 & 2.98 & -81.0 & 0.57 & R4 \\
\hline 2008.55 & 0.496 & 3.03 & -106.7 & 1.11 & R3 \\
\hline 2008.55 & 0.400 & 6.32 & -78.6 & 1.35 & $\mathrm{Y}$ \\
\hline 2008.55 & 0.556 & 9.23 & -64.1 & 4.86 & $\mathrm{Z}$ \\
\hline 2008.58 & 7.499 & 0.00 & 0.0 & 0.00 & K \\
\hline 2008.58 & 2.192 & 0.19 & -106.1 & 0.03 & $\mathrm{C}$ \\
\hline 2008.58 & 0.485 & 0.64 & -97.6 & 0.47 & A \\
\hline 2008.58 & 0.711 & 1.40 & -95.5 & 0.61 & B \\
\hline 2008.58 & 0.103 & 2.34 & -39.0 & 0.86 & R1 \\
\hline 2008.58 & 0.124 & 2.83 & -63.2 & 0.94 & R2 \\
\hline 2008.58 & 0.126 & 3.02 & -81.7 & 0.47 & $\mathrm{R} 4$ \\
\hline 2008.58 & 0.512 & 3.06 & -106.5 & 1.17 & R3 \\
\hline 2008.58 & 0.382 & 6.33 & -78.6 & 1.32 & $\mathrm{Y}$ \\
\hline 2008.58 & 0.598 & 9.28 & -64.0 & 5.24 & $\mathrm{Z}$ \\
\hline 2008.60 & 7.677 & 0.00 & 0.0 & 0.00 & K \\
\hline 2008.60 & 2.406 & 0.17 & -103.5 & 0.07 & $\mathrm{C}$ \\
\hline 2008.60 & 0.402 & 0.60 & -97.7 & 0.42 & A \\
\hline 2008.60 & 0.757 & 1.39 & -96.2 & 0.66 & B \\
\hline 2008.60 & 0.127 & 2.37 & -40.5 & 1.17 & R1 \\
\hline 2008.60 & 0.184 & 3.00 & -83.0 & 0.60 & R4 \\
\hline 2008.60 & 0.081 & 3.04 & -60.0 & 0.81 & $\mathrm{R} 2$ \\
\hline 2008.60 & 0.429 & 3.10 & -108.6 & 0.97 & R3 \\
\hline 2008.60 & 0.384 & 6.32 & -78.2 & 1.32 & $\mathrm{Y}$ \\
\hline 2008.60 & 0.557 & 9.40 & -63.6 & 4.98 & $\mathrm{Z}$ \\
\hline 2008.76 & 9.659 & 0.00 & 0.0 & 0.04 & K \\
\hline 2008.76 & 2.743 & 0.18 & -110.1 & 0.13 & $\mathrm{C}$ \\
\hline 2008.76 & 0.390 & 0.71 & -101.6 & 0.41 & A \\
\hline 2008.76 & 0.461 & 1.41 & -97.2 & 0.51 & B \\
\hline 2008.76 & 0.289 & 2.28 & -56.7 & 2.00 & R1 \\
\hline 2008.76 & 0.540 & 2.96 & -97.2 & 1.59 & R4 \\
\hline
\end{tabular}

Table 2. continued.

\begin{tabular}{|c|c|c|c|c|c|}
\hline Epoch & $\begin{array}{l}\mathrm{FD} \\
{[\mathrm{Jy}]} \\
(2)\end{array}$ & $\begin{array}{c}\text { C Sep } \\
\text { [mas] } \\
\text { (3) }\end{array}$ & $\begin{array}{c}\text { PA } \\
{[\mathrm{deg}]} \\
(4)\end{array}$ & $\begin{array}{c}\text { MA } \\
{[\mathrm{mas}]} \\
(5)\end{array}$ & Id \\
\hline 2008.76 & 0.132 & 3.17 & -113.6 & 0.53 & R3 \\
\hline 2008.76 & 0.386 & 6.34 & -78.6 & 1.38 & $\mathrm{Y}$ \\
\hline 2008.76 & 0.453 & 8.86 & -64.5 & 2.80 & $\mathrm{Z}$ \\
\hline 2009.15 & 5.328 & 0.00 & 0.0 & 0.08 & K \\
\hline 2009.15 & 1.947 & 0.22 & -110.7 & 0.16 & $\mathrm{C}$ \\
\hline 2009.15 & 0.503 & 0.68 & -99.3 & 0.45 & A \\
\hline 2009.15 & 0.414 & 1.46 & -98.0 & 0.57 & B \\
\hline 2009.15 & 0.262 & 2.43 & -53.9 & 1.65 & R1 \\
\hline 2009.15 & 0.107 & 3.14 & -79.4 & 0.48 & $\mathrm{R} 2$ \\
\hline 2009.15 & 0.550 & 3.17 & -105.3 & 1.25 & R3 \\
\hline 2009.15 & 0.416 & 6.32 & -78.5 & 1.42 & $\mathrm{Y}$ \\
\hline 2009.15 & 0.464 & 8.92 & -65.1 & 2.79 & $\mathrm{Z}$ \\
\hline 2009.48 & 3.024 & 0.00 & 0.0 & 0.12 & K \\
\hline 2009.48 & 1.223 & 0.28 & -107.1 & 0.14 & $\mathrm{C}$ \\
\hline 2009.48 & 0.519 & 0.63 & -97.2 & 0.43 & A \\
\hline 2009.48 & 0.351 & 1.41 & -96.8 & 0.63 & B \\
\hline 2009.48 & 0.156 & 2.40 & -42.7 & 1.21 & $\mathrm{R} 1$ \\
\hline 2009.48 & 0.188 & 3.16 & -77.4 & 0.88 & R2 \\
\hline 2009.48 & 0.520 & 3.18 & -104.6 & 1.23 & R3 \\
\hline 2009.48 & 0.407 & 6.29 & -78.1 & 1.48 & Y \\
\hline 2009.48 & 0.510 & 8.86 & -64.6 & 3.14 & $\mathrm{Z}$ \\
\hline 2009.73 & 2.814 & 0.00 & 0.0 & 0.13 & $\mathrm{~K}$ \\
\hline 2009.73 & 1.119 & 0.33 & -105.0 & 0.18 & $\mathrm{C}$ \\
\hline 2009.73 & 0.476 & 0.67 & -97.6 & 0.36 & A \\
\hline 2009.73 & 0.264 & 1.48 & -96.6 & 0.53 & B \\
\hline 2009.73 & 0.164 & 2.35 & -45.3 & 1.44 & R1 \\
\hline 2009.73 & 0.156 & 3.10 & -73.6 & 0.99 & $\mathrm{R} 2$ \\
\hline 2009.73 & 0.569 & 3.25 & -103.0 & 1.39 & R3 \\
\hline 2009.73 & 0.398 & 6.32 & -78.0 & 1.42 & $\mathrm{Y}$ \\
\hline 2009.73 & 0.522 & 8.83 & -64.4 & 3.20 & $\mathrm{Z}$ \\
\hline 2009.92 & 5.197 & 0.00 & 0.0 & 0.08 & K \\
\hline 2009.92 & 0.738 & 0.25 & -99.1 & 0.18 & $\mathrm{C}$ \\
\hline 2009.92 & 1.007 & 0.54 & -101.6 & 0.37 & A \\
\hline 2009.92 & 0.350 & 1.41 & -96.0 & 0.81 & B \\
\hline 2009.92 & 0.153 & 2.52 & -46.7 & 1.22 & R1 \\
\hline 2009.92 & 0.122 & 3.15 & -72.9 & 0.91 & R2 \\
\hline 2009.92 & 0.555 & 3.35 & -101.8 & 1.37 & R3 \\
\hline 2009.92 & 0.419 & 6.38 & -77.7 & 1.45 & $\mathrm{Y}$ \\
\hline 2009.92 & 0.483 & 8.91 & -64.5 & 2.85 & $\mathrm{Z}$ \\
\hline 2010.05 & 9.032 & 0.00 & 0.0 & 0.09 & $\mathrm{~K}$ \\
\hline 2010.05 & 0.458 & 0.26 & -101.8 & 0.16 & $\mathrm{C}$ \\
\hline 2010.05 & 0.967 & 0.56 & -105.1 & 0.34 & A \\
\hline 2010.05 & 0.302 & 1.36 & -95.5 & 0.75 & B \\
\hline 2010.05 & 0.163 & 2.60 & -51.4 & 1.16 & R1 \\
\hline 2010.05 & 0.078 & 3.20 & -76.8 & 0.49 & $\mathrm{R} 2$ \\
\hline 2010.05 & 0.529 & 3.35 & -102.4 & 1.44 & R3 \\
\hline 2010.05 & 0.367 & 6.34 & -77.7 & 1.40 & $\mathrm{Y}$ \\
\hline 2010.05 & 0.169 & 8.09 & -67.5 & 1.55 & $\mathrm{Z}$ \\
\hline 2010.05 & 0.299 & 9.86 & -62.8 & 3.04 & $\mathrm{Z}$ \\
\hline 2010.14 & 1.920 & 0.00 & 0.0 & 0.00 & $\mathrm{~K}$ \\
\hline 2010.14 & 12.036 & 0.11 & -81.9 & 0.11 & D \\
\hline 2010.14 & 0.955 & 0.56 & -101.4 & 0.25 & $\mathrm{C}$ \\
\hline 2010.14 & 0.357 & 0.83 & -96.6 & 0.34 & A \\
\hline 2010.14 & 0.211 & 1.58 & -96.4 & 0.59 & B \\
\hline 2010.14 & 0.264 & 2.85 & -60.7 & 1.78 & R1 \\
\hline 2010.14 & 0.027 & 3.41 & -82.5 & 0.37 & R2 \\
\hline 2010.14 & 0.555 & 3.45 & -102.1 & 1.46 & R3 \\
\hline 2010.14 & 0.387 & 6.47 & -78.0 & 1.39 & $\mathrm{Y}$ \\
\hline 2010.14 & 0.114 & 8.06 & -67.6 & 1.23 & $\mathrm{Z}$ \\
\hline 2010.14 & 0.373 & 9.70 & -63.6 & 3.21 & $\mathrm{Z}$ \\
\hline 2010.47 & 5.276 & 0.00 & 0.0 & 0.00 & $\mathrm{~K}$ \\
\hline
\end{tabular}


Table 2. continued.

\begin{tabular}{|c|c|c|c|c|c|}
\hline Epoch & $\begin{array}{l}\text { FD } \\
\text { [Jy] } \\
(2)\end{array}$ & $\begin{array}{l}\text { C Sep } \\
\text { [mas] }\end{array}$ & $\begin{array}{c}\text { PA } \\
{[\mathrm{deg}]}\end{array}$ & $\begin{array}{c}\text { MA } \\
{[\mathrm{mas}]}\end{array}$ & Id \\
\hline 2010.47 & 16.303 & 0.13 & -82.7 & 0.15 & $\mathrm{D}$ \\
\hline 2010.47 & 0.675 & 0.59 & -98.3 & 0.12 & $\mathrm{C}$ \\
\hline 2010.47 & 0.838 & 0.71 & -98.4 & 0.41 & A \\
\hline 2010.47 & 0.163 & 1.54 & -101.4 & 0.62 & B \\
\hline 2010.47 & 0.296 & 2.58 & -61.7 & 1.92 & $\mathrm{R} 1$ \\
\hline 2010.47 & 0.515 & 3.48 & -101.1 & 1.50 & R3 \\
\hline 2010.47 & 0.382 & 6.37 & -77.6 & 1.45 & $\mathrm{Y}$ \\
\hline 2010.47 & 0.126 & 8.12 & -68.1 & 1.20 & $\mathrm{Z}$ \\
\hline 2010.47 & 0.362 & 9.64 & -63.5 & 3.12 & $\mathrm{Z}$ \\
\hline 2010.65 & 5.956 & 0.00 & 0.0 & 0.00 & $\mathrm{~K}$ \\
\hline 2010.65 & 18.320 & 0.17 & -87.9 & 0.12 & $\mathrm{D}$ \\
\hline 2010.65 & 0.854 & 0.50 & -97.6 & 0.13 & $\mathrm{C}$ \\
\hline 2010.65 & 0.883 & 0.74 & -101.0 & 0.20 & A \\
\hline 2010.65 & 0.127 & 1.45 & -96.2 & 0.55 & B \\
\hline 2010.65 & 0.421 & 2.60 & -71.6 & 2.47 & $\mathrm{R} 1$ \\
\hline 2010.65 & 0.427 & 3.58 & -102.7 & 1.33 & R3 \\
\hline 2010.65 & 0.383 & 6.40 & -77.9 & 1.44 & $\mathrm{Y}$ \\
\hline 2010.65 & 0.417 & 8.95 & -65.1 & 2.70 & $\mathrm{Z}$ \\
\hline 2010.74 & 5.852 & 0.00 & 0.0 & 0.00 & $\mathrm{~K}$ \\
\hline 2010.74 & 14.762 & 0.17 & -94.0 & 0.11 & D \\
\hline 2010.74 & 1.901 & 0.44 & -100.0 & 0.30 & $\mathrm{C}$ \\
\hline 2010.74 & 0.705 & 0.79 & -101.7 & 0.16 & A \\
\hline 2010.74 & 0.620 & 2.10 & -78.8 & 2.60 & $\mathrm{x}$ \\
\hline 2010.74 & 0.351 & 3.69 & -101.5 & 1.23 & R3 \\
\hline 2010.74 & 0.358 & 6.44 & -77.9 & 1.43 & Y \\
\hline 2010.74 & 0.527 & 8.94 & -64.8 & 3.41 & $\mathrm{Z}$ \\
\hline 2010.89 & 7.148 & 0.00 & 0.0 & 0.00 & $\mathrm{~K}$ \\
\hline 2010.89 & 14.092 & 0.19 & -87.6 & 0.14 & D \\
\hline 2010.89 & 1.953 & 0.46 & -97.2 & 0.33 & $\mathrm{C}$ \\
\hline 2010.89 & 0.649 & 0.81 & -104.3 & 0.24 & A \\
\hline 2010.89 & 0.614 & 2.09 & -77.5 & 2.79 & $\mathrm{x}$ \\
\hline 2010.89 & 0.311 & 3.66 & -102.7 & 0.95 & R3 \\
\hline 2010.89 & 0.419 & 6.42 & -77.6 & 1.62 & Y \\
\hline 2010.89 & 0.417 & 9.17 & -63.3 & 2.73 & $\mathrm{Z}$ \\
\hline 2011.28 & 10.907 & 0.00 & 0.0 & 0.11 & $\mathrm{~K}$ \\
\hline 2011.28 & 8.136 & 0.27 & -71.4 & 0.19 & D \\
\hline 2011.28 & 1.333 & 0.53 & -88.9 & 0.00 & $\mathrm{C}$ \\
\hline 2011.28 & 0.846 & 0.71 & -105.7 & 0.38 & A \\
\hline 2011.28 & 0.041 & 1.52 & -103.9 & 0.22 & B \\
\hline 2011.28 & 0.536 & 2.98 & -81.0 & 2.80 & $\mathrm{x}$ \\
\hline 2011.28 & 0.209 & 3.65 & -102.6 & 0.87 & R3 \\
\hline 2011.28 & 0.275 & 6.47 & -77.4 & 1.16 & $\mathrm{Y}$ \\
\hline 2011.28 & 0.591 & 8.68 & -64.5 & 3.38 & $\mathrm{Z}$ \\
\hline 2011.48 & 8.353 & 0.00 & 0.0 & 0.18 & $\mathrm{~K}$ \\
\hline 2011.48 & 6.338 & 0.33 & -77.7 & 0.20 & D \\
\hline 2011.48 & 0.143 & 0.53 & 47.9 & 0.02 & $\mathrm{x}$ \\
\hline 2011.48 & 1.047 & 0.59 & -100.2 & 0.00 & $\mathrm{C}$ \\
\hline 2011.48 & 0.449 & 0.82 & -100.7 & 0.59 & A \\
\hline 2011.48 & 0.498 & 2.53 & -82.3 & 2.85 & $\mathrm{x}$ \\
\hline 2011.48 & 0.219 & 3.79 & -101.6 & 0.81 & R3 \\
\hline 2011.48 & 0.299 & 6.35 & -77.3 & 1.27 & $\mathrm{Y}$ \\
\hline 2011.48 & 0.617 & 8.66 & -62.8 & 3.87 & $\mathrm{Z}$ \\
\hline
\end{tabular}



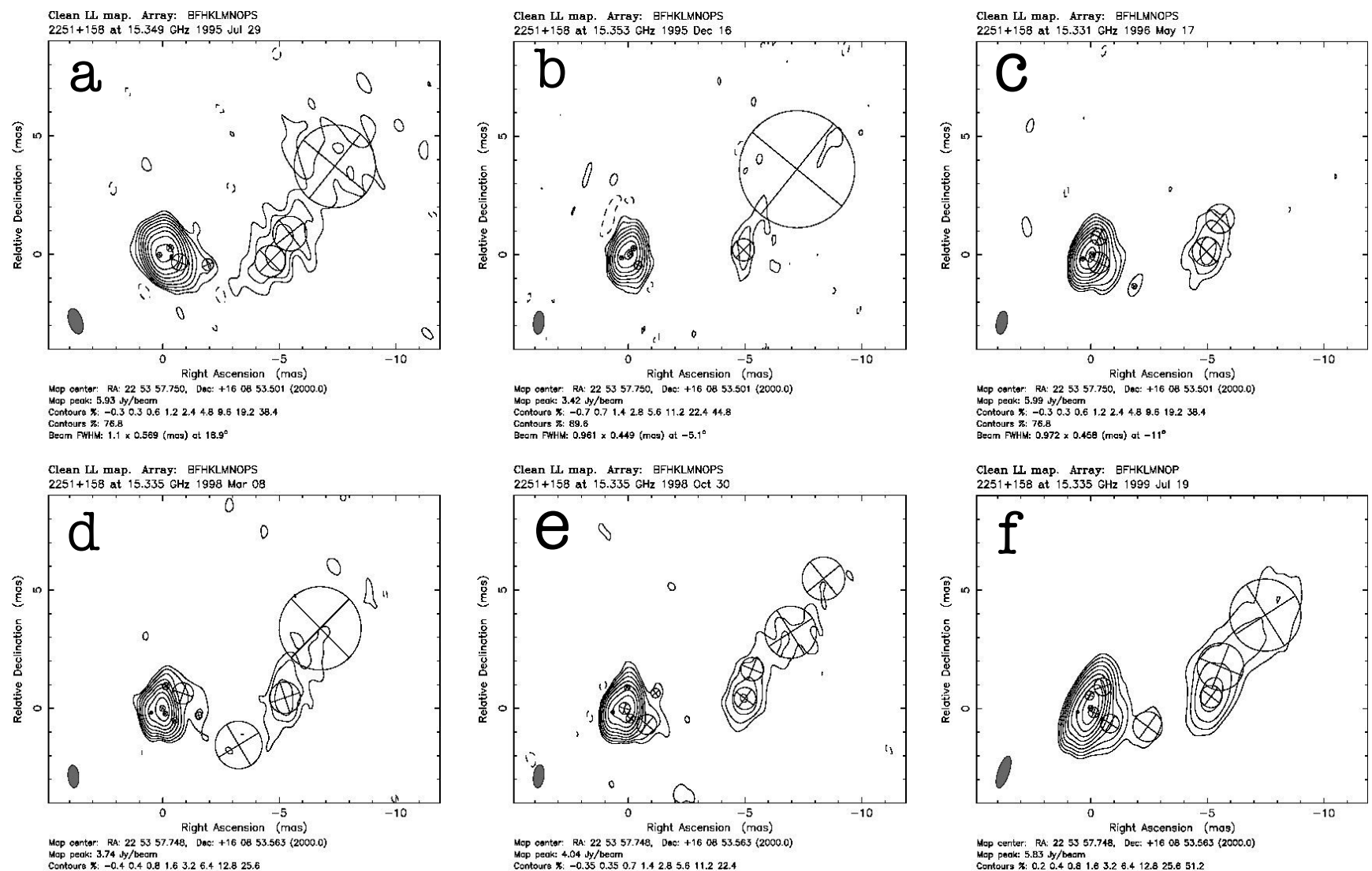

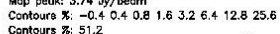

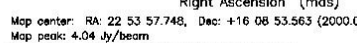

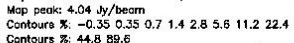

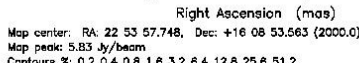

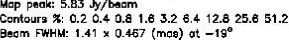

Clean I map. Array: BFHKLMNOPS
$2251+158$ at 15.165 GHz 2000 Jun 27
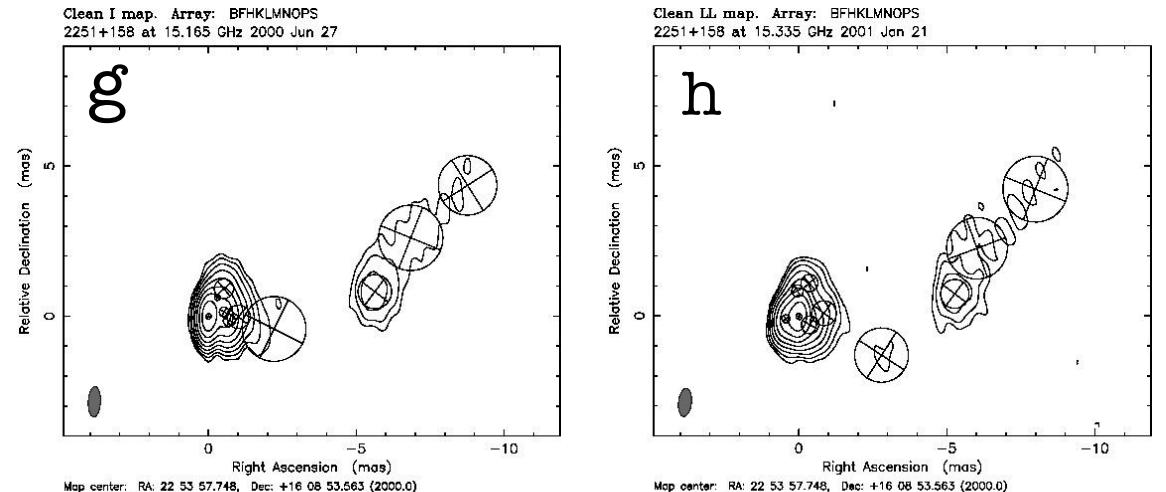

Clean I map. Array: BFHKLMNOPS
$2251+158$ at $15.365 \mathrm{GHz} 2003$ Mar 30

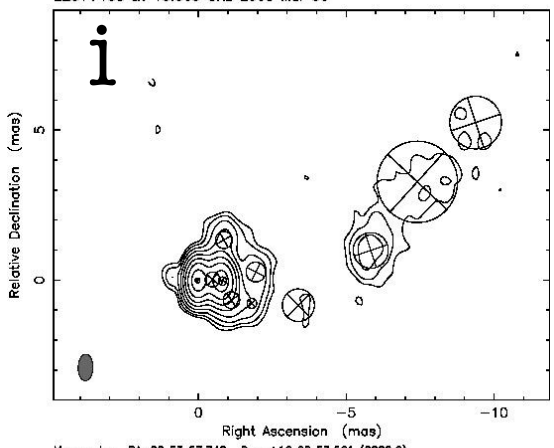

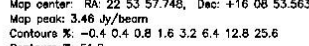

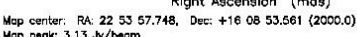

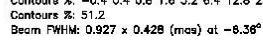

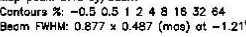

Clean I map. Array: BFKLNOPHM
$2251+158$ at $15.3655 \mathrm{GHz} 2005$ Apr 21

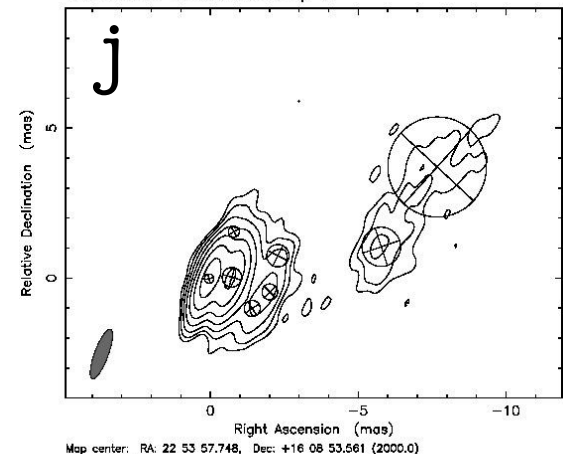

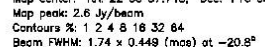
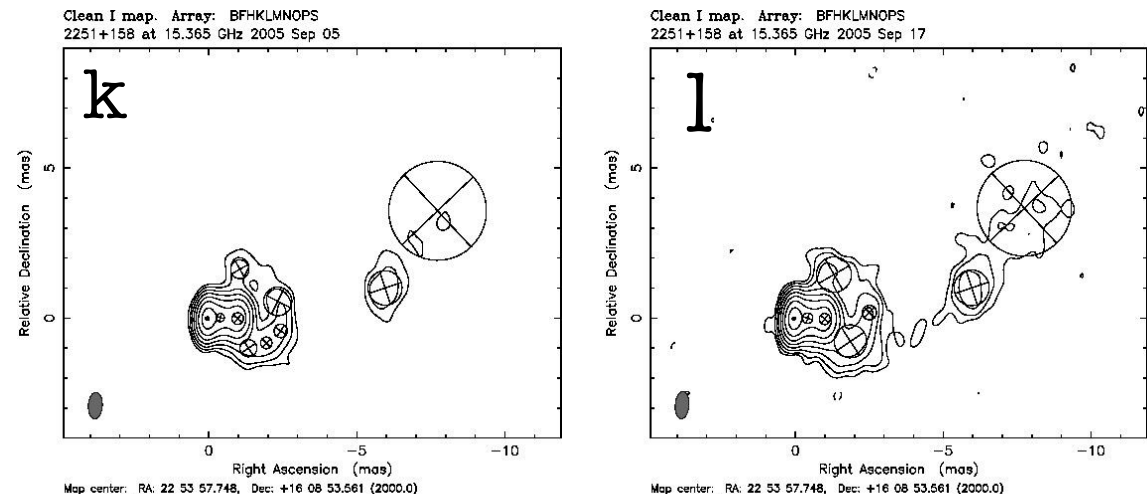

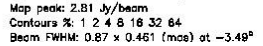

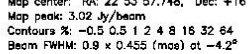

Fig. 3. Images of $2251+158$ observed at $15 \mathrm{GHz}$ for epochs $1995 \mathrm{Jul} .29$ to $2005 \mathrm{Sep}$. 17. Gaussian model fit components are superimposed for each image. The restoring beam of each image is presented as a shaded ellipse. 

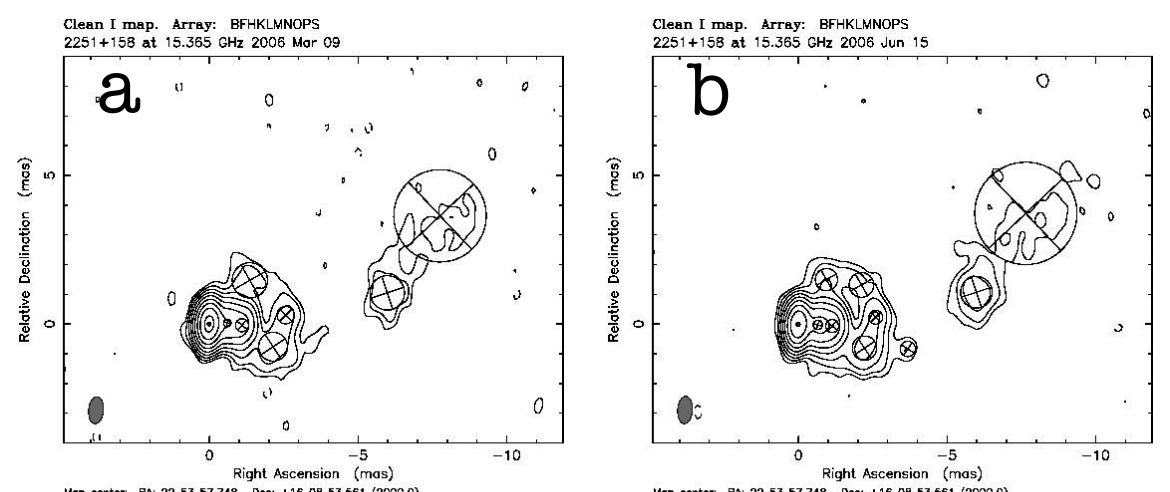

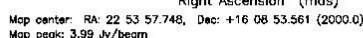

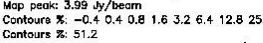

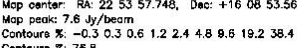

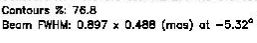

Clean I map. Array: BFHKLMNOPS
$2251+158$ at
15.365
GHz 2008 Jun 25

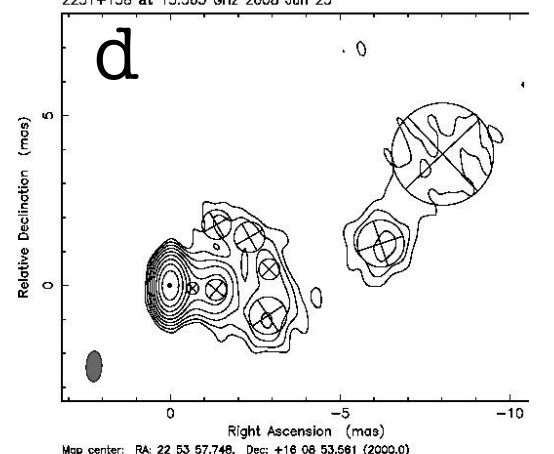

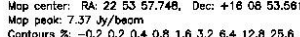

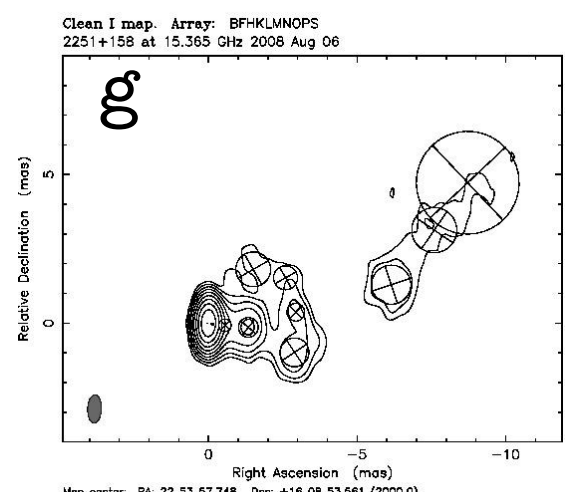

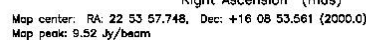

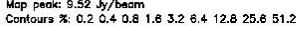

Clean I map. Array: BFFKL MOPSN
$2251+158$ at 15.357 chtz 2009 Uun 25

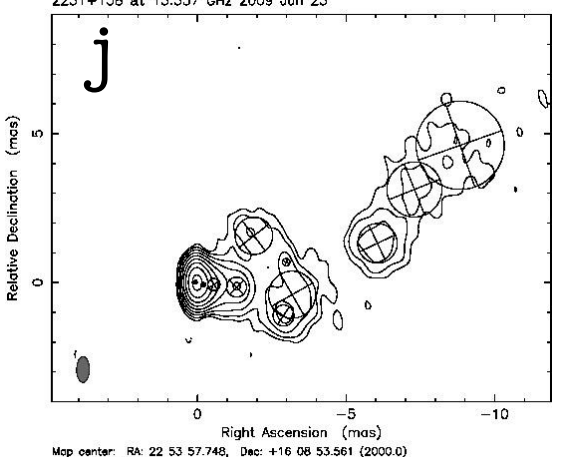

Mop cantiter RA: 225357.748 , Das: +16 ab 53.561 (20000

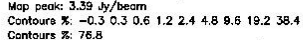

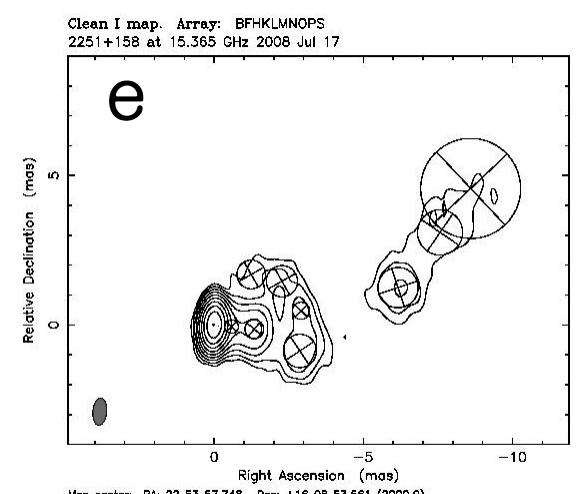

Map center. RA: 225357.748, Dese +160853.561 (20000)

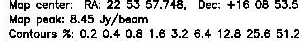

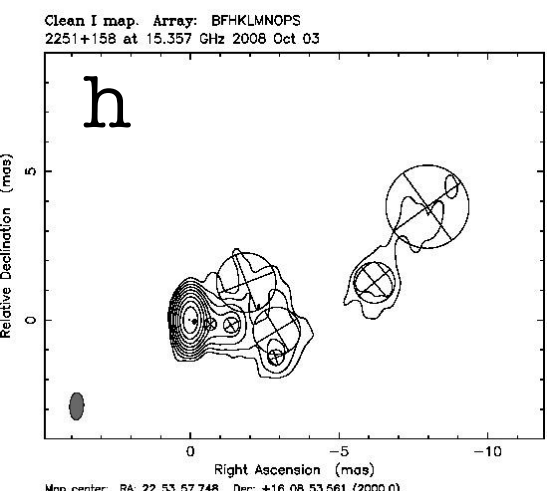

Mop center: RA: 225357.748, Deci +160853.561 (20000.0

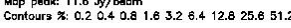

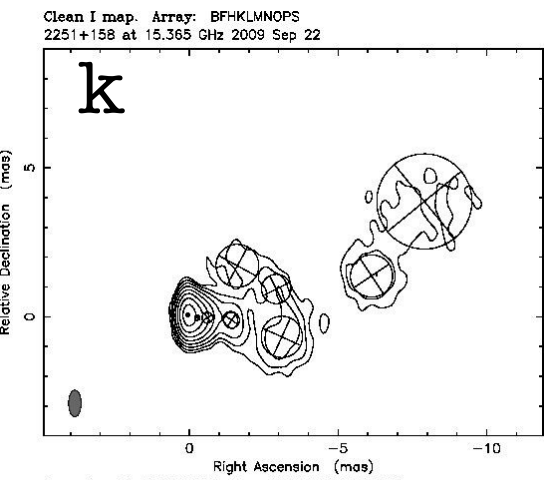

Mop center: RA: 225357.748 . Dee: +160853.561 (20000.0

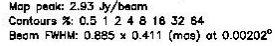

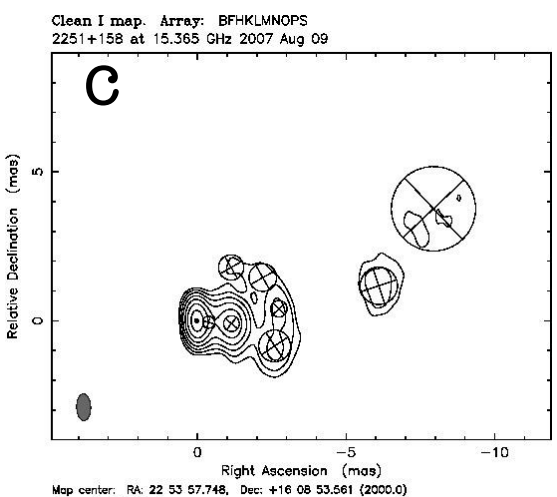

Mop certer: RA. 225357.748 , Dev 160853.561 (2000.0)

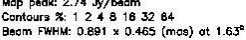

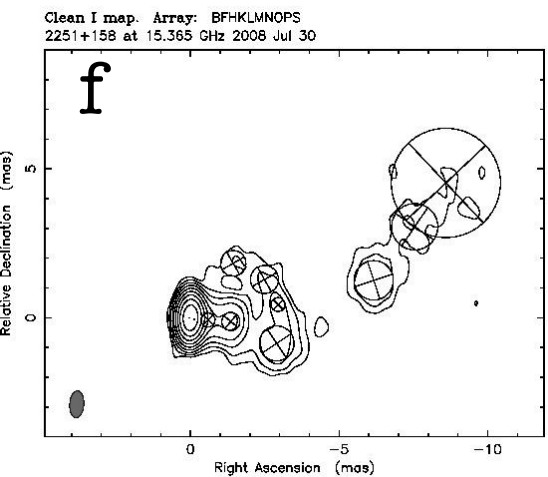

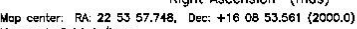

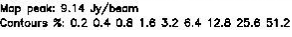

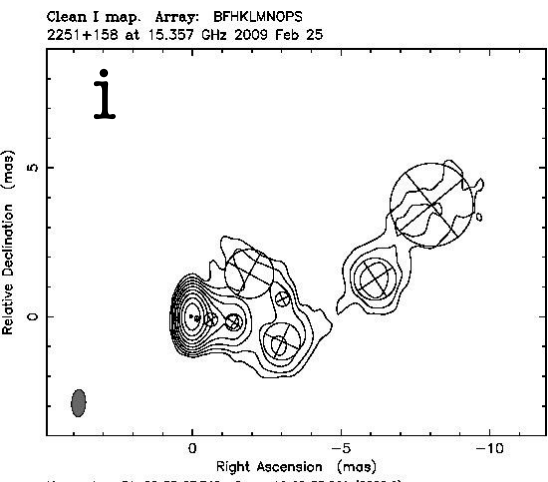

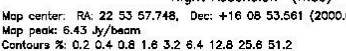

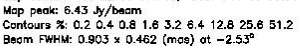

Clean I map. Arrag: BFHKLMNOPS
$2251+158$ at $15.365 \mathrm{GHz} 2009$ Dec 03

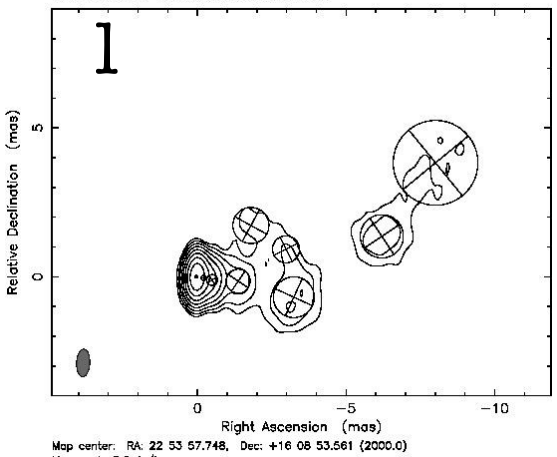

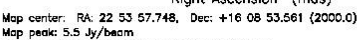

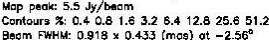

Fig. 4. Images of 2251+158 observed at $15 \mathrm{GHz}$ for epochs 2006 Mar. 09 to $2009 \mathrm{Dec}$. 03. Gaussian model fit components are superimposed for each image. The restoring beam of each image is presented as a shaded ellipse. 
Clean I map. Array: BFKLMNOPS
$2251+158$ at $15.365 \mathrm{GHz} 2010$ Jan 18

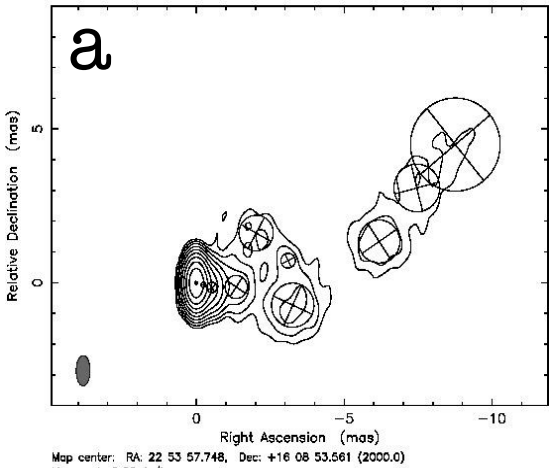

Contours 20.20 .20 .40 .81 .632 .26 .412 .825 .651 .2

Clean I map. Array: BFHKLMNOPS

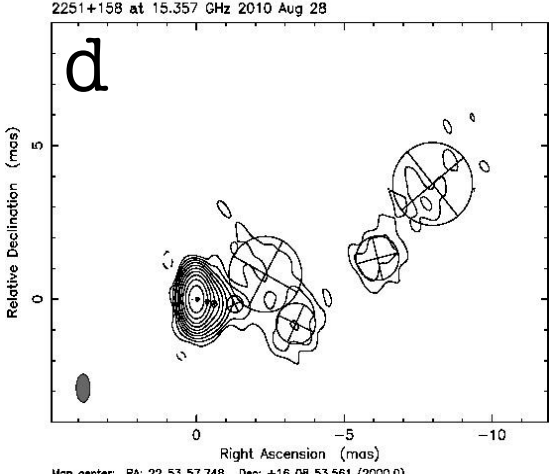

Mop cantar. RA: 225357.748 . Das: +16 a8 53.561 (2000.0)

Contours 2.4 - 0.1 ly/beam 0.10 .20 .40 .81 .53 .26 .412 .8

Gontcurs \%: 25.651 .2 .20 .42 (mag) at $0.63^{\circ}$

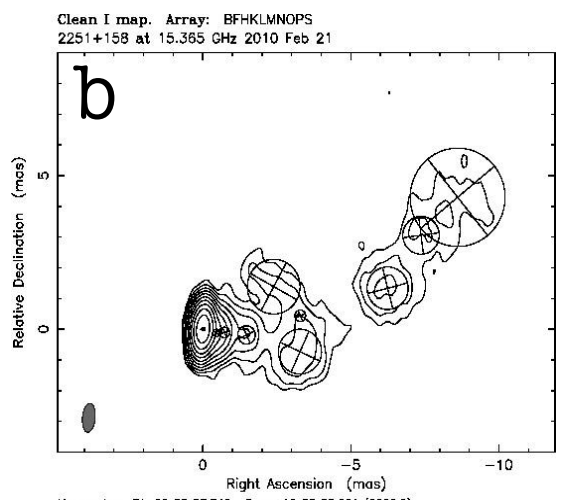

Map cantar: RA 2253 57.748, Dases +160853.561 (2000.0)

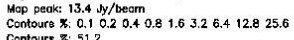

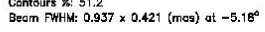

Clean I map. Array: BFHKLMNOPS

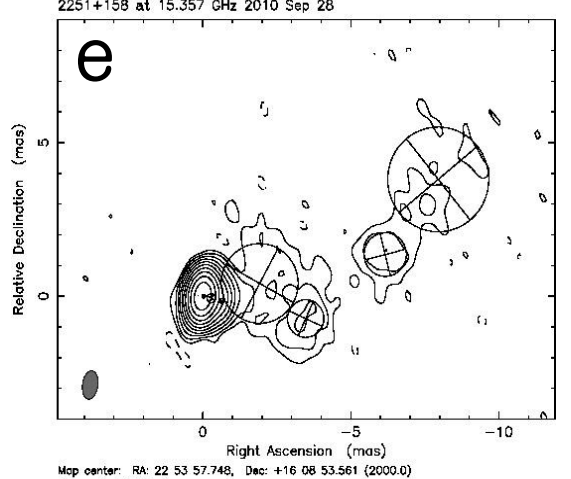

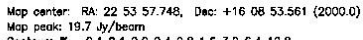

Contourar X: -0.10 .10 .20 .40 .81 .63 .26 .412 .8$

Contours $z: 25.651 .2 .20 .409$ (mag) at $-8.46^{\circ}$
Clean I map. Array: BFHKLMNOPS

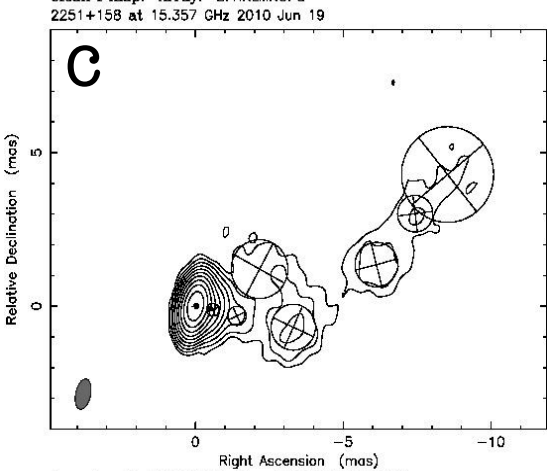

Right Ascension (mas)
Mop contar: RA 225357748 Deci +160853.561 (20000.0)

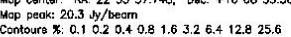

Beam

Clean I map. Array: BFHKLMNOPS

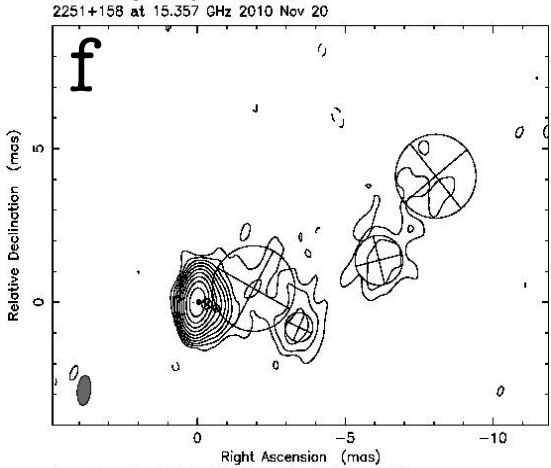

Mop cantar: RA 2253 57.748. Dase: +160853.561 (2000.0)

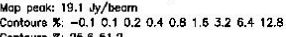

Conteurs $3: 25.551 .2$

Fig. 5. Images of 2251+158 observed at $15 \mathrm{GHz}$ for epochs 2010 Jan. 18 to 2010 Nov. 20. Gaussian model fit components are superimposed for each image. The restoring beam of each image is presented as a shaded ellipse. 
A\&A 557, A37 (2013)

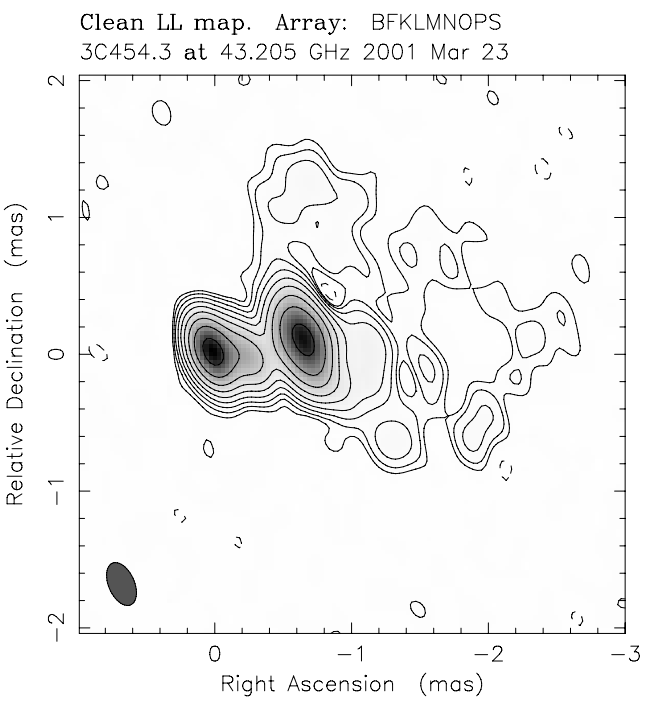

Mao center: RA: 2253 57.748. Dec: +16 0853.563 (2000.0)

Map peak: $1.45 \mathrm{Jy} /$ beam

Contours \%: $-0.3 \quad 0.3 \quad 0.61 .22 .4 \quad 4.8 \quad 9.619 .2 \quad 38.4$

Contours \%: 76.8

Beam FWHM: $0.332 \times 0.188$ (mas) at $23.8^{\circ}$

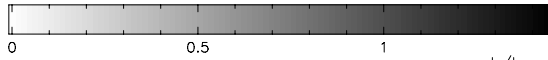

Clean I map. Array: BF $\angle$ NOPM

Jy/beam

36454.3 at $43.221 \mathrm{GHz} 2005 \mathrm{Moy} 22$

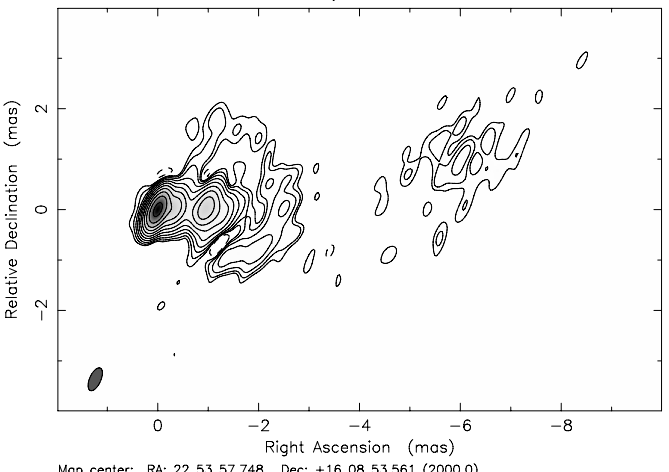

Nop center: RA: 2253557.748 , Dec: +1

Contours: \%: -0.0750 .0750 .150 .30 .61 .22 .44 .8$

Beam FWHM: $0.486 \times 0.221$ (mas) at -24.2

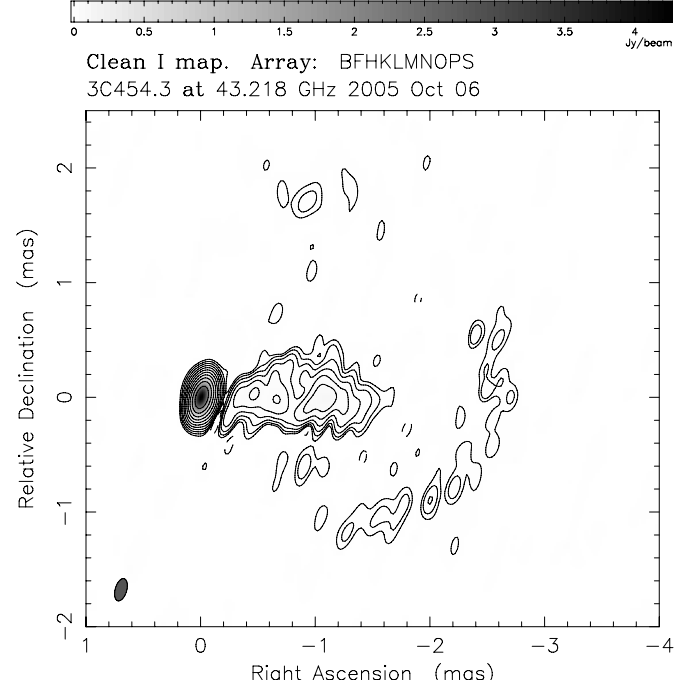

Map center: RA: 2253 57.748, Dec. + 160853.561 (2000.0)

Map peak: 8.66 Jy/beam

Contours \%: $-0.05 \quad 0.050 .10 .20 .40 .81 .63 .26 .4$

Contours

Beam FWHM: $0.2 \times 0.1$ (mas) at $-16^{\circ}$

$\longrightarrow$

Fig. 6. Images of $2251+158$ at $43 \mathrm{GHz}$. 
S. Britzen et al.: An arc-like structure in $2251+158$

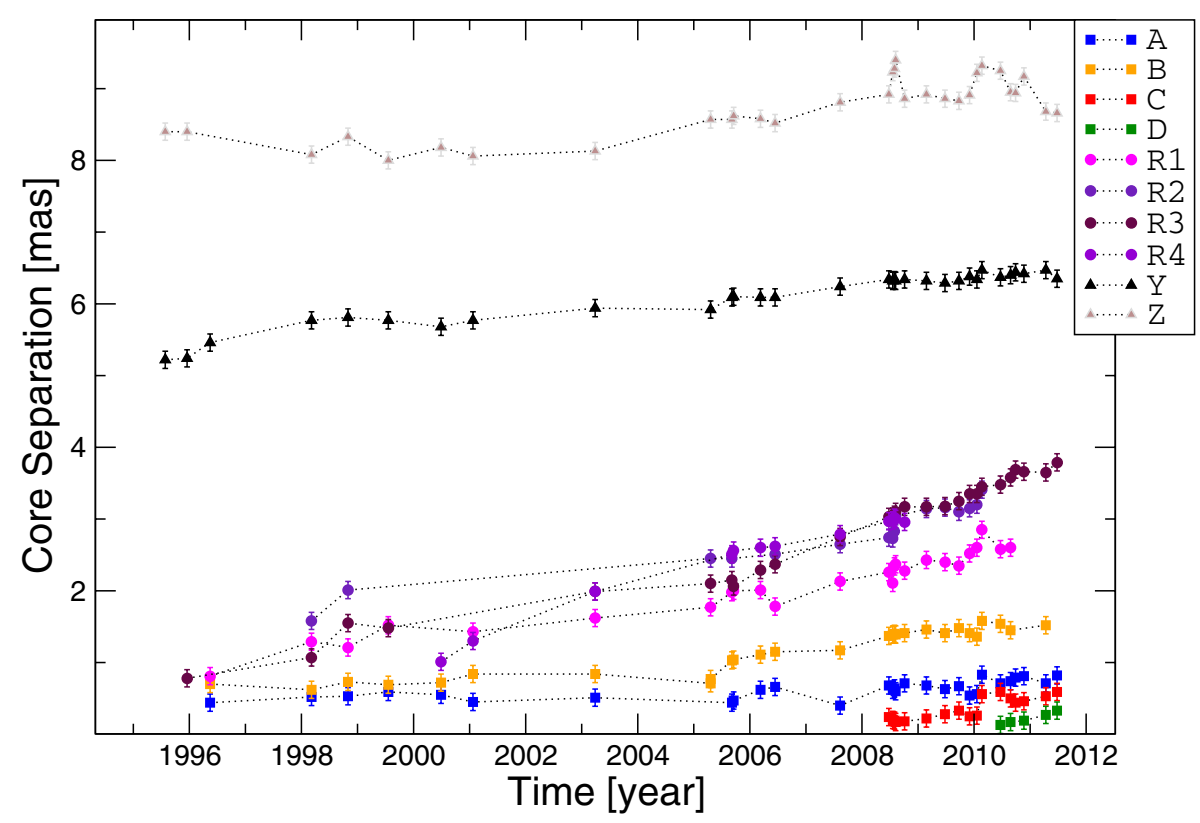

Fig. 7. Separation from the core as function of time shown for all reliably identified components in the VLBI jet of 2251+158. 
The inner jet components ( $<1.8$ mas)
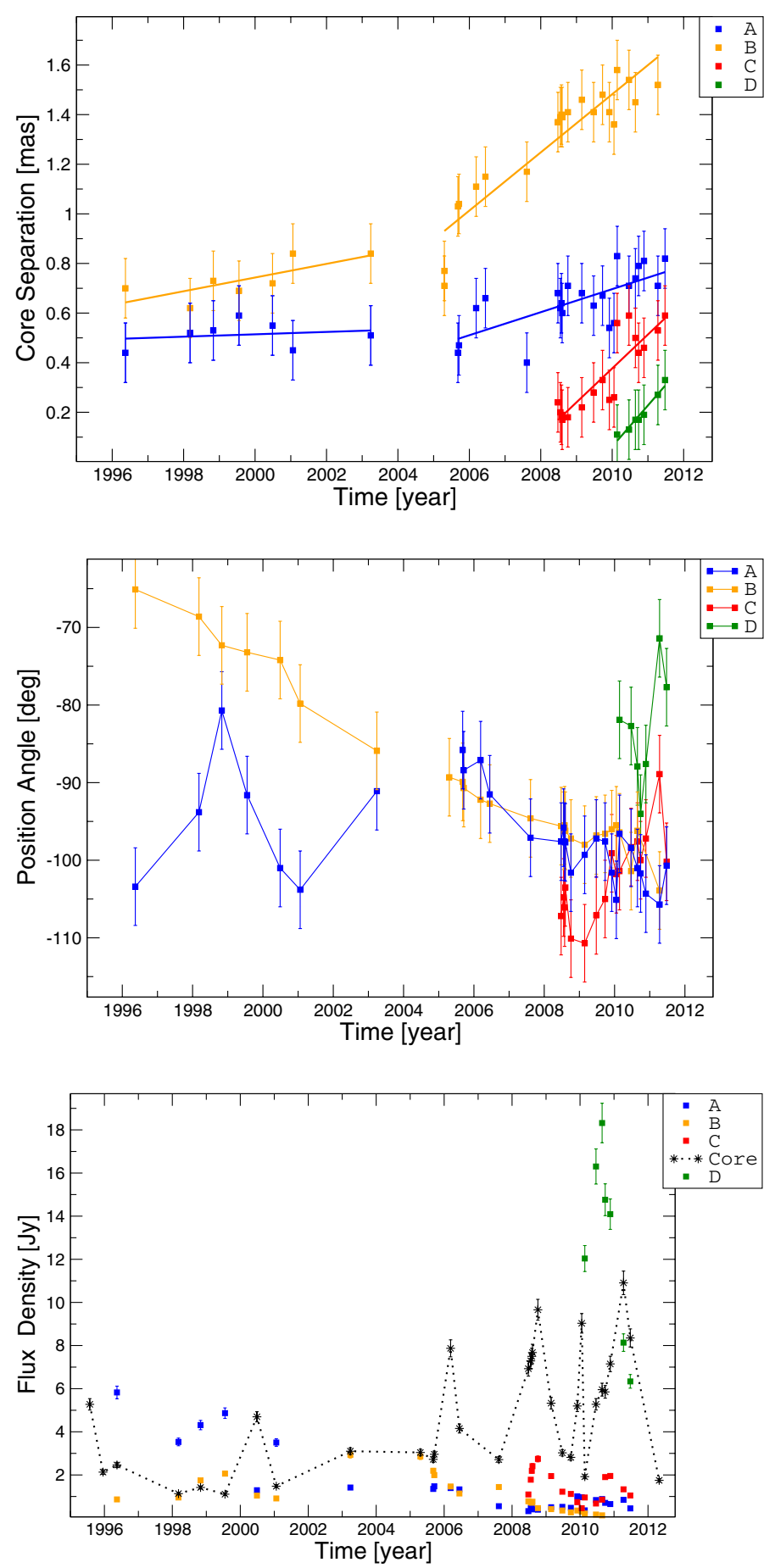

Fig. 8. Inner jet components and their properties. From top to bottom: distance from the core as function of time, position angle of the component relative to the core as function of time, and flux density as function of time. Please note that the bottom image also displays the flux density evolution of the core.
Components of the arc $(<4$ mas distance from core $)$
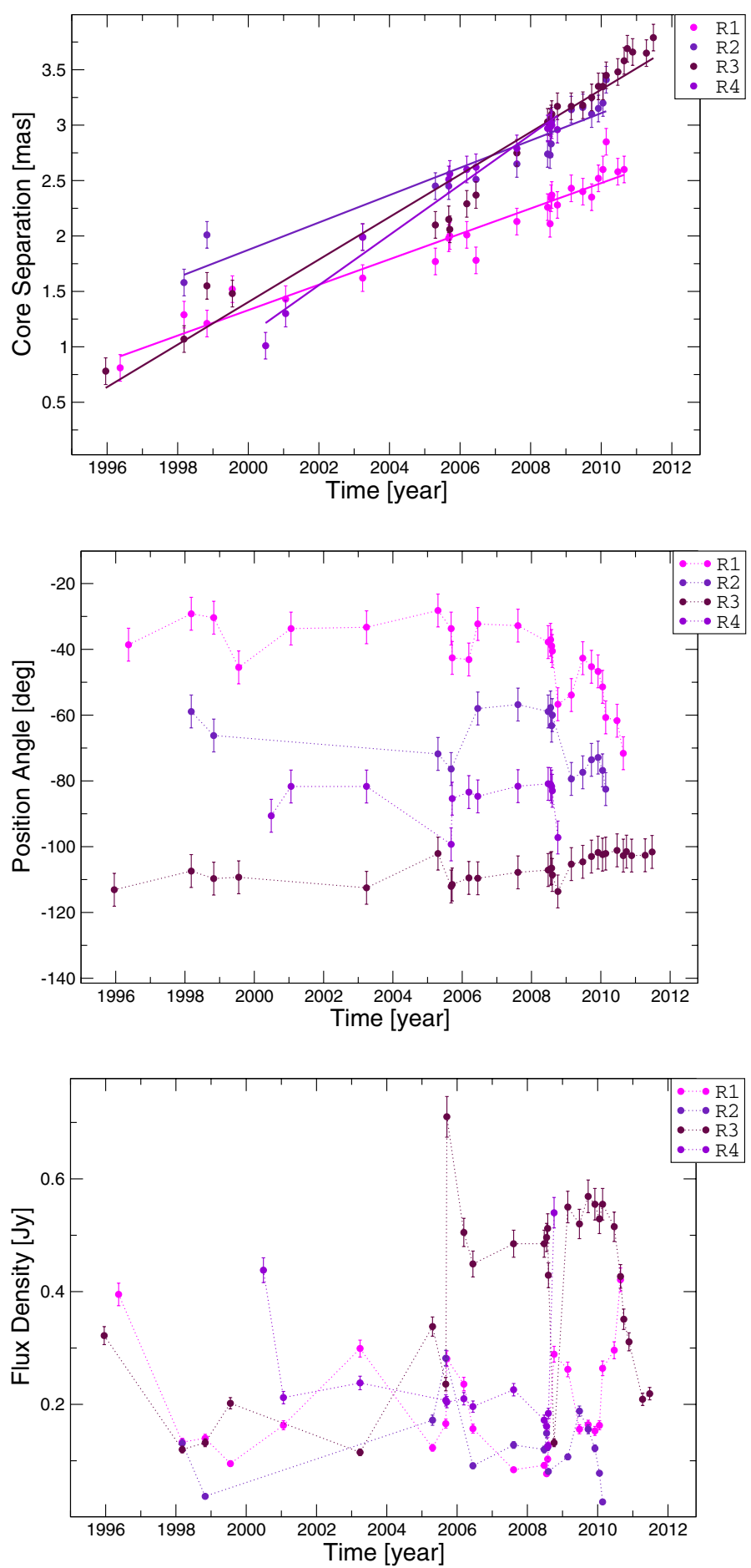

Fig. 9. Components of the arc-like structure and their properties. From top to bottom: distance from the core as function of time, position angle of the component relative to the core as function of time, and flux density as function of time. 
Outer jet components (> 5mas)
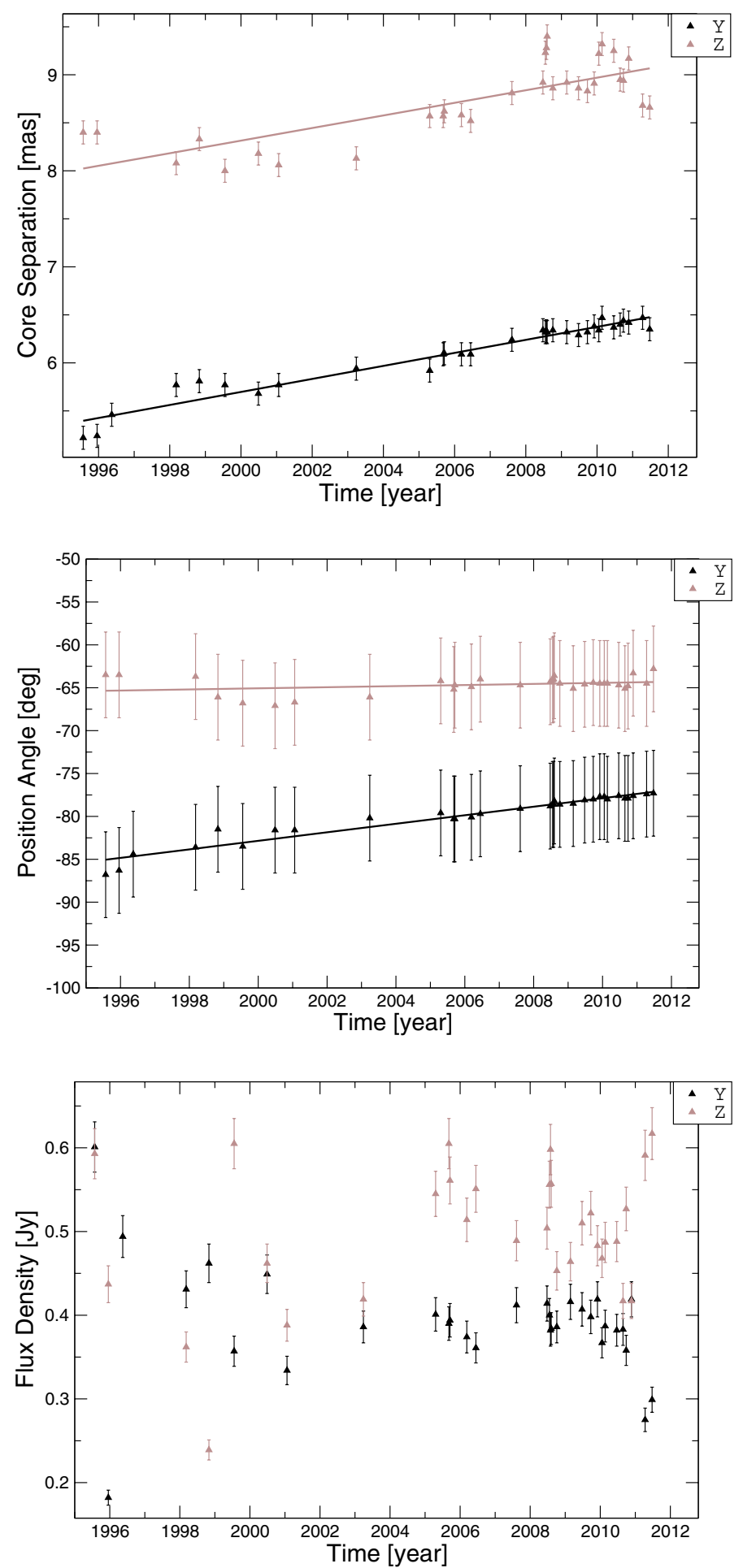

Fig. 10. Outer jet components and their properties. From top to bottom: distance from the core as function of time, position angle of the component relative to the core as function of time, and flux density as function of time.

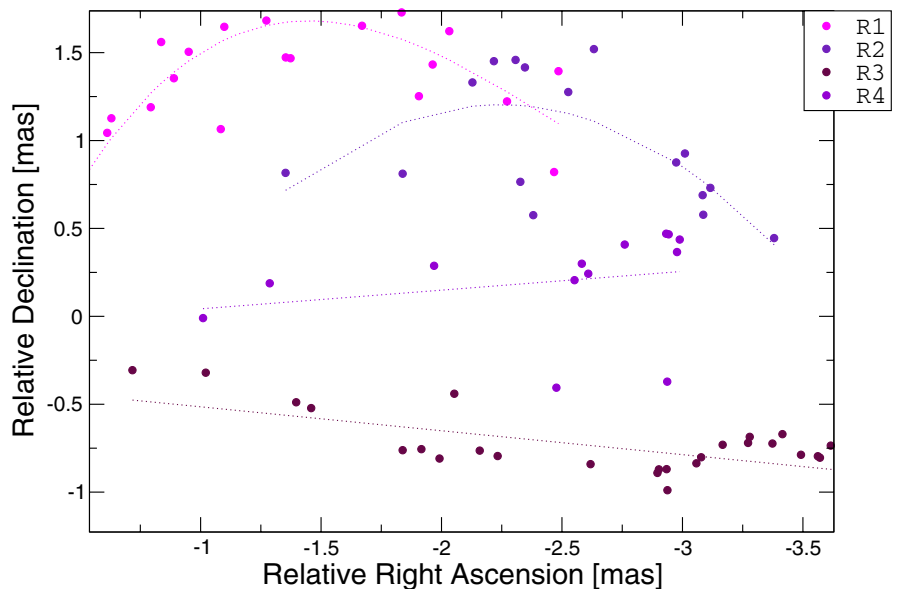

Fig. 11. Coordinates of the arc-components R1, R2, R3, and R4. Dotted lines indicate a cubic regression model to $\mathrm{R} 1$, quadratic regression model to R2, and a linear regression model to R3 and R4. The fits are meant to visualize the trends, not to represent an optimum fit to the data.
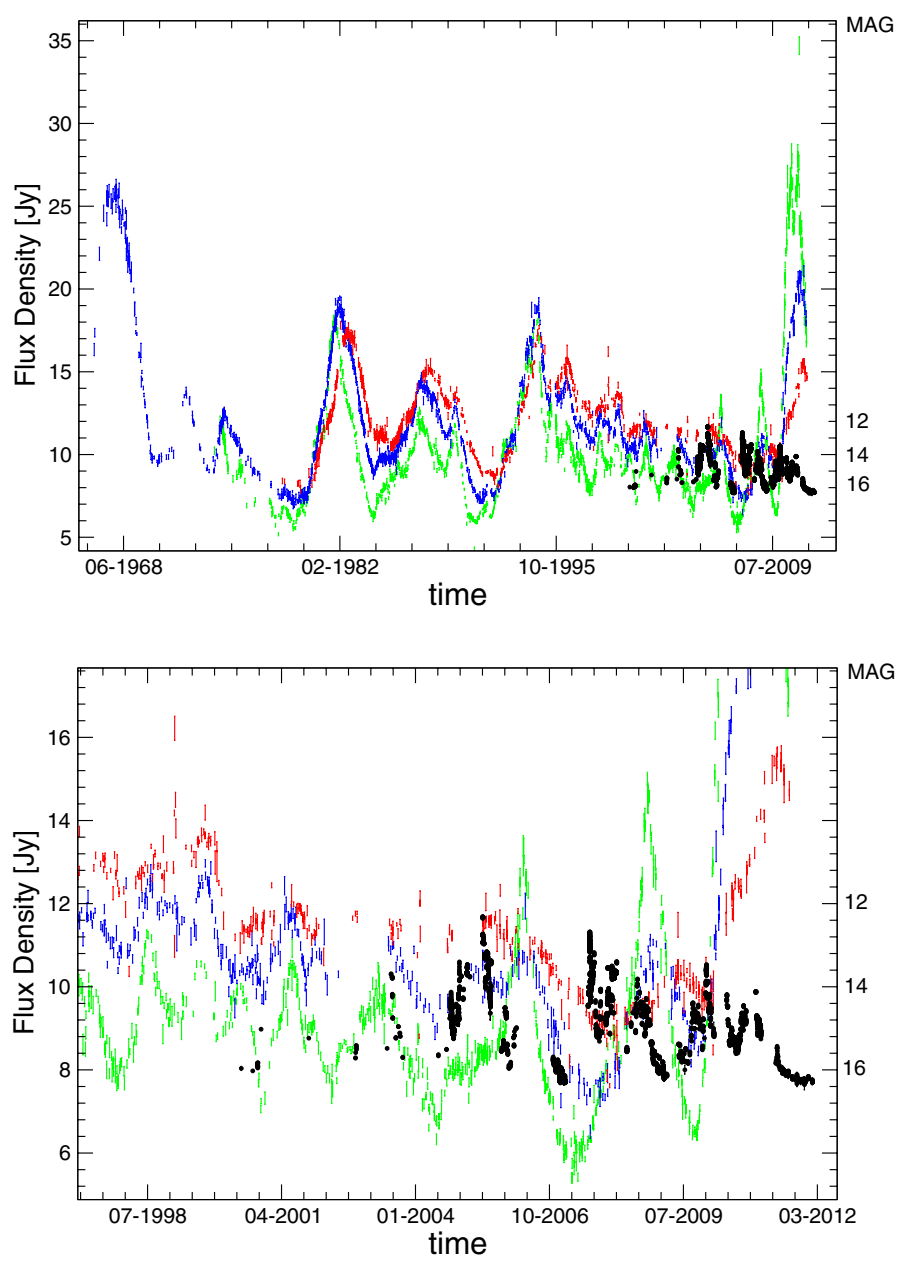

Fig. 12. Flux density monitoring. The top panel shows the single-dish radio flux densities as observed within the Michigan monitoring program. Flux densities observed at 4.8 (red), 8.0 (blue), and $14.5 \mathrm{GHz}$ (green) are shown. Superimposed is the $R$-band light-curve (black data points) obtained with the Abastumani optical telescope. Note that the two $y$-axes are labeled differently (the left $y$-axis describes the radio flux density, the right $y$-axis the optical magnitude). Please also note that the optical data cover the time between 2000 Jun. 03 and 2012 Feb. 02. The plot at the bottom is an expansion of the plot at the top. 